\title{
Photometric metallicity map of the Small Magellanic Cloud
}

\author{
S. Choudhury, ${ }^{1 \star}$ A. Subramaniam, ${ }^{2 \star}$ A. A. Cole ${ }^{3}$ and Y.-J. Sohn ${ }^{1,4}$ \\ ${ }^{1}$ Yonsei University Observatory, Seoul 120-749 Republic of Korea \\ ${ }^{2}$ Indian Institute of Astrophysics, 2 B Koramangala, Bangalore 560034, India \\ ${ }^{3}$ School of Natural Sciences, University of Tasmania, Private Bag 37, Hobart, Tasmania 7001, Australia \\ ${ }^{4}$ Department of Astronomy, Yonsei University, Seoul 03722, Republic of Korea
}

Accepted 2018 January 8. Received 2017 December 15; in original form 2017 August 21

\begin{abstract}
We have created an estimated metallicity map of the Small Magellanic Cloud (SMC) using the Magellanic Cloud Photometric Survey (MCPS) and Optical Gravitational Lensing Experiment (OGLE III) photometric data. This is a first of its kind map of metallicity up to a radius of $\sim 2.5^{\circ}$. We identify the Red Giant Branch (RGB) in the $V,(V-I)$ colour-magnitude diagrams of small sub-regions of varying sizes in both data sets. We use the slope of the RGB as an indicator of the average metallicity of a sub-region and calibrate the RGB slope to metallicity using available spectroscopic data for selected sub-regions. The average metallicity of the SMC is found to be $[\mathrm{Fe} / \mathrm{H}]=-0.94 \mathrm{dex}(\sigma[\mathrm{Fe} / \mathrm{H}]=0.09)$ from OGLE III and $[\mathrm{Fe} / \mathrm{H}]=-0.95 \mathrm{dex}$ $(\sigma[\mathrm{Fe} / \mathrm{H}]=0.08)$ from MCPS. We confirm a shallow but significant metallicity gradient within the inner SMC up to a radius of $2.5^{\circ}\left(-0.045 \pm 0.004\right.$ to $\left.-0.067 \pm 0.006 \mathrm{dex} \mathrm{deg}^{-1}\right)$.
\end{abstract}

Key words: stars: abundances-Hertzsprung-Russell and colour-magnitude diagramsgalaxies: abundances-Local Group-Magellanic Clouds.

\section{INTRODUCTION}

The Magellanic Clouds (MCs), comprising the Large Magellanic Cloud (LMC; $d \sim 50 \mathrm{kpc}$ ) and the Small Magellanic Cloud (SMC; $d \sim 60 \mathrm{kpc}$ ), are the closest pair of interacting galaxies to the Milky Way (MW). The LMC is a late-type spiral galaxy seen nearly faceon, whereas the structure of the SMC is not yet well-established. The gas and young stars are supposedly distributed in an highly inclined disc of the SMC, whereas the old- and intermediate-age stellar populations are found to be distributed in a regular, smooth spheroidal/ellipsoidal component (Subramanian \& Subramaniam 2015). This canonical vision of the structure of the SMC is being challenged by several recent works on variable stars, both old (RR Lyrae; e.g. Jacyszyn-Dobrzeniecka et al. 2017; Muraveva et al. 2018) and young (Classical Cepheids; e.g. Jacyszyn-Dobrzeniecka et al. 2016; Scowcroft et al. 2016; Ripepi et al. 2017). Optically, the appearance of the SMC is dominated by an elongated bar-like structure in the north-east and south-west directions, from which a prominent Wing extends to the east, in a direction that joins the Magellanic Bridge (MB) and the LMC (Nidever et al. 2011). The relative proximity of these galaxies has allowed detailed analyses of the metallicity of individual field and cluster stars to probe their star formation and chemical enrichment history.

The MCs are embedded within a common envelope of neutral hydrogen, indicating that these two galaxies are interacting with each other. It was also believed that the MCs have had interactions with the MW as well as amongst each other (Murai \& Fujimoto

\footnotetext{
* E-mail: samyaday.choudhury@gmail.com (SC); purni@iiap.res.in (AS)
}

1980; Tanaka 1981; Fujimoto \& Murai 1984; Gardiner, Sawa \& Fujimoto 1994; Westerlund 1997). However, recent measurements of their proper motion (Kallivayalil et al. 2006a,b, 2013) suggest that they are approaching the MW for the first time (Besla et al. 2007, 2012). The MCs are metal poor $(Z \approx 0.008$ for LMC and 0.004 for the SMC), gas rich, and have active ongoing star formation, possibly triggered by interactions between themselves and/or interactions with the Galaxy. However, the mutual interaction between the Clouds, rather than the interaction with the MW, is fundamental in shaping their star formation history and metallicity gradients (MGs; Cioni 2009).

The MG within a galaxy can give indication of its formation and chemical evolution. This is especially interesting in the case of the SMC, which supposedly is dominated by its dynamical interaction history with the LMC and the MW. These interactions can trigger star formation and produce changes in the chemical evolution of the galaxy over a certain period of time. During a collapse scenario gas is accreted and falls into the centre of the galaxy, where further star formation takes place thus enriching the pre-existing gas (Cioni 2009). Stars may also form during the accretion process at large distances from the centre. The bar, disc, and halo components of a galaxy's potential can play a role in its chemical evolution. Also, dynamical interaction between galaxies and the accretion of satellites can alter the distribution of gas, modulating its chemical evolution and population gradients. Thus, accurate measurement of the MG is important to interpret the formation and evolution mechanisms.

The existence of an MG in the SMC has been a long-standing debate. Previous studies have used star clusters (Piatti et al. 2007a,b; Parisi et al. 2009, 2015) as well as field stars (Carrera et al. 2008; Cioni 2009; Piatti 2012; Dobbie et al. 2014b; Parisi et al. 2016) 
within the SMC in order to understand its MG. These studies included the use of spectroscopic as well as photometric data.

Cioni (2009) used a photometric technique to estimate the MG of field asymptotic giant branch (AGB) stars within the SMC, by comparing the ratio of carbon-rich ( $\mathrm{C}$ type) to oxygen-rich ( $\mathrm{M}$ type) AGBs. Using this $\mathrm{C} / \mathrm{M}$ ratio as an indicator of $[\mathrm{Fe} / \mathrm{H}]$ abundance, the authors estimated an almost constant metallicity $(-1.25 \pm 0.01 \mathrm{dex})$ to a galactocentric distance of about $12 \mathrm{kpc}$. Although these authors could cover a large area of the SMC, their indicators (AGB) and calibrators [Red Giant Branch (RGB) stars] were different, and the $\mathrm{C} / \mathrm{M}$ ratio is potentially susceptible to age effects. Parisi et al. (2015) used a sample of about $36 \mathrm{SMC}$ star clusters by combining samples from (Parisi et al. 2009) and other previous studies. They found a bimodality in the metallicity distribution of the SMC clusters with potential peaks at -1.1 and -0.8 dex but no strong MG. Piatti (2012) in an attempt to understand the age-metallicity relation in the SMC, analysed about 3.3 million field stars distributed throughout the entire main body using Washington photometric data. The author reported that the field stars do not possess gradients in age and metallicity. Also, the stellar populations formed since $\sim 2 \mathrm{Gyr}$ ago are more metal rich than $[\mathrm{Fe} / \mathrm{H}] \sim-0.8$ dex and are confined to the innermost region (semimajor axis $\leq 1^{\circ}$ ).

In work based on variable stars, Haschke, Grebel \& Duffau (2012) analysed the Optical Gravitational Lensing Experiment III (OGLE III) data of the SMC to obtain a mean metallicity of $-1.70 \pm 0.27$ dex based on Fourier decomposition of $I$-band light curves of 1831 RR Lyraes. Their value was in very good agreement with earlier spectroscopic and photometric metallicities of RR Lyraes obtained by Butler, Demarque \& Smith (1982) and Kapakos, Hatzidimitriou \& Soszyński (2011), respectively. Haschke et al. (2012), however, did not detect any MG for these older populations within the SMC. Also, Deb et al. (2015) did not detect any MG from the analysis of more than 1000 RR Lyrae stars using OGLE III data. Thus, all the above studies provided no evidence for any MG within the SMC.

On a different front there are studies that provide an evidence for an MG. Carrera et al. (2008) estimated a mean $[\mathrm{Fe} / \mathrm{H}] \sim-1.0 \mathrm{dex}$ within the inner SMC, using CaT spectroscopy of over 350 RGB stars in 13 fields distributed in different positions in the SMC (ranging from $1^{\circ}$ to $4^{\circ}$ from its centre). The authors found that this mean metallicity decreases as one moves towards the outermost regions from centre. The most extensive spectroscopic study of RGB stars within the SMC has been carried out by Dobbie et al. (2014a,b). Dobbie et al. (2014b) used CaT spectroscopy of about 3000 stars within inner $5^{\circ}$ of the SMC and confirmed a me$\operatorname{dian}[\mathrm{Fe} / \mathrm{H}]=-0.99 \pm 0.01$ with clear evidence for an abundance gradient of $-0.075 \pm 0.011 \mathrm{dex} \mathrm{deg}^{-1}$. Later on Parisi et al. (2016) created an enlarged sample $(\sim 750)$ of RGB stars using their previous study (Parisi et al. 2010) and estimated a median metallicity of $[\mathrm{Fe} / \mathrm{H}] \sim-0.97 \pm 0.01$ and detected a gradient of $-0.08 \pm 0.02 \mathrm{dex} \mathrm{deg}^{-1}$ within the inner $4^{\circ}$. This value of MG was similar to that estimated by Dobbie et al. (2014b).

Rubele et al. (2015) attempted to understand the star formation history across the main body and Wing of the SMC $\left(14 \mathrm{deg}^{2}\right)$ using near-infrared data (VISTA survey of the MCs) by employing a colour-magnitude diagram (CMD) reconstruction method. The authors analysed about 120 sub-regions, each covering $21 \operatorname{arcmin} \times$ $21.5 \mathrm{arcmin}$, and presented the spatial distribution of mean metallicity for populations of three different ages $(\sim 800 \mathrm{Myr}, 1.3 \mathrm{Gyr}$, and $2 \mathrm{Gyr}$ ). They found mean metallicity between $-0.4 \geq[\mathrm{Fe} / \mathrm{H}] \geq$ -0.85 dex for each age bin. However, the authors do not estimate the MG within the SMC. Kapakos \& Hatzidimitriou (2012) per- formed Fourier decomposition analysis of 8- and 13-yr $V$-band light curves of 454 fundamental-mode RR Lyrae variables from the OGLE III Catalogue of Variable Stars. The authors estimated an average metal abundance of $-1.69 \pm 0.41 \mathrm{dex}$, with a tentative MG $\left(-0.013 \pm 0.007 \mathrm{dex} \mathrm{kpc}^{-1}\right)$ that indicated increasing metal abundance towards the dynamical centre of the SMC.

In a previous attempt to understand the metallicity variation within the LMC, Choudhury, Subramaniam \& Cole (2016) (hereafter Paper I) created a first of its kind, high-spatial resolution metallicity map with RGB stars as the tool, using the Magellanic Cloud Photometric Survey (MCPS) and OGLE III photometric data. The RGB is identified in the $V,(V-I)$ CMDs of small sub-regions of varying sizes in both data sets. The slope of the RGB is used as an indicator of the mean metallicity of a sub-region, and it is calibrated to metallicity using spectroscopic data for field and cluster RGB stars in selected sub-regions. The study reconfirms that reliable photometric metallicity estimates can cover a large area of the galaxy, unlike the spectroscopic method, which can over only relatively small areas. The photometric method can bring out the overall distribution, variation, and global average of metallicity. It also can identify regions that might show large deviation with respect to the mean value.

A high-spatial metallicity map showing the metallicity trend across the inner SMC is still unavailable. Also, it is seen that there has been less consensus over the nature of the MG within the SMC. Thus, we require a study using spatially extensive and homogeneous data sets to make significant advances. In this study, we extend the techniques developed in Paper I to the SMC with similar data sets to estimate a first of its kind high-spatial resolution metallicity map for the smaller Cloud.

The paper is organized in the following way. In Section 2, we describe the data (OGLE III and MCPS) used in this study. The OGLE III analysis, metallicity maps and results are presented in Section 3, whereas those corresponding to the MCPS data are presented in Section 4. Section 5 describes the error analysis corresponding to our estimations. The discussion related to our study are presented in Section 6. We summarize the conclusions in Section 7.

\section{DATA}

For this study, we have used two large photometric surveys of the SMC, OGLE III, and the MCPS. The OGLE III survey covered a total area of about $14 \mathrm{deg}^{2}$ (Udalski et al. 2008) and presented calibrated $V$ - and $I$-band photometry of about 6.2 million stars. OGLE III covers the central region as well as the eastern and western regions of the inner SMC till a radius of $\sim 2.5^{\circ}$ from the SMC centre. The MCPS provided the catalogue for over 5 million stars in the SMC, within the central $18 \mathrm{deg}^{2}$ of the SMC (Zaritsky et al. 2002). The survey presented the photometric as well as extinction maps in $U, B, V$, and $I$ passbands for the SMC. The MCPS survey is of relatively lower resolution ( $\left.0.70 \mathrm{arcsec} \mathrm{pixel}^{-1}\right)$ compared to the OGLE III ( $\left.0.26 \operatorname{arcsec~pixel}^{-1}\right)$ survey. We only consider stars with photometric error less than $0.15 \mathrm{mag}$ in the $V$ and $I$ passbands in both data sets. The MCPS has more coverage of the northern and southern regions of the central SMC, whereas the OGLE III survey has more coverage of the eastern and western regions. The two surveys thus complement each other in terms of area covered.

\section{OGLE III ANALYSIS}

We use the slope of the RGB in the CMD of a small region in the galaxy as an indicator of the mean metallicity of the region. A 
region in a galaxy with metal-rich stars is expected to have a shallower RGB slope when compared to a relatively metal-poor region. The dependence of slope of the RGB on metallicity is well known and has been studied primarily for homogeneous populations, i.e. star clusters (Da Costa \& Armandroff 1990; Kuchinski et al. 1995). In Paper I, we applied this concept for the first time to field stars, which are heterogeneous with respect to age and metallicity. The RGB slope of a field region will correspond to the metallicity of the dominant RGB population of the region. Section 3 of Paper I describes in detail the salient features of the robust process developed to identify and estimate the RGB slope consistently within the CMDs of small regions within the galaxy, independent of differential reddening.

The OGLE III observed region is binned into 656 small regions, each of dimension $(8.88 \times 8.88) \operatorname{arcmin}^{2}$ in RA and Dec. We identify the RGB by (i) segregating it from other evolutionary stages, (ii) using the location of Red Clump (RC) stars to locate the base of the $\mathrm{RGB}$, and (iii) estimating the slope of the RGB in CMDs of all these small regions. According to Subramanian \& Subramaniam (2009), most of the SMC regions have a large number of RC stars and their distribution can be well identified in the CMDs of the regions. The use of the peak of the RC as RGB base allows us to uniquely and consistently define a location in the RGB and identify it uniformly in CMDs of all the regions. Thus, even if this is not the actual base of the RGB, the part of RGB used for slope estimation is made uniform for all location.

Similar to Paper I, the RGB slope estimation procedure was tested and verified for a large number of SMC sub-regions before validating. We briefly mention the primary steps involved in the process (for detailed steps we direct the readers to section 3 of Paper I):

(1) Excluding the main sequence (MS) and including mainly the evolved portion of the CMD with stars having $0.5<(V-I) \leq$ $2.5 \mathrm{mag}$ and $12.0 \leq V<20.0 \mathrm{mag}$ (Fig. 1a). (2) Constructing a density diagram to identify the location of RC stars and assume the peak $(V-I)$ and $V$ of the RC distribution as the base of the RGB (Fig. 1b). This location changes with the reddening, along with the location of the RGB, and is used to identify the RGB similarly in all the regions. (3) Removing bluer and fainter bins with respect to the $\mathrm{RC}$ peak by giving a cut in colour and magnitude corresponding to the RC peak (Fig. 1c). The extracted part of the CMD is dominated by RGB stars, but contaminated with some other evolutionary phases (AGBs, etc.) to a lesser extent. (4) Finally, in order to identify the RGB unambiguously and to reduce the scatter in the RGB and eliminate the other evolutionary phases, we consider only those colour-magnitude bins that contain a minimum number of three stars. The chosen criterion eliminates the brighter part of the RGB, typically sampling the RGB from the RC peak up to 2 mag brighter in most of the regions. As shown in Fig. 1(d), the selected part of the RGB thus appears to be more or less a straight line, without the curved brighter part. These bins, thus representing the RGB, are fitted with a straight line and the slope is estimated using the method of least-squares fit ( $3 \sigma$ clipping with a single iteration).

The total number of stars in a particular region is given by $N$. We define $N_{p}$, as the number of CMD bins (with number of stars in each bin $\geq 3$ ) representing the RGB. The slope of RGB is negative, but we denote it by its absolute value as |slope|, and the error in slope as $\sigma_{\text {slope }}$. We also express the correlation coefficient by its absolute value, as $r$. Thus, we have calculated $N_{p}$, |slope|, $\sigma_{\text {slope }}$, and $r$ for each of the regions.
The above method is found to work consistently for most of the regions, except when there is a large variation in reddening and/or multiple dominant stellar population. The central regions that have higher stellar density show a scatter in the RC and RGB, thus providing poor estimation of slope (lower $r$ ). In order to probe the effect on the fit due to overpopulated CMDs, Fig. 2 shows a plot of $N_{p}$ versus $N$. The figure shows that as $N$ increases, $N_{p}$ also increases, suggesting that, in general, the denser areas have well-populated RGBs. In Fig. 3, the correlation between $N_{p}$ and $r$ is shown, where it is seen that the regions with high $N_{p}$ (i.e. correspondingly higher $N$ ) have lower $r(<0.50)$, suggesting a poor fit/estimation of slope. This is similar to figs 2 and 3 of Paper I, where we note that adopting similar areas for all regions across the galaxy leads to poor estimation of slopes for regions that have high stellar density. Inspection of such regions suggests that the broad RGB in such regions are likely caused by small-scale variation in reddening and/or multiple dominant population.

We further showed in Paper I that finer sub-division of regions based on stellar density can help get rid of this problem. To achieve this, we adopted six binning criteria for the OGLE III regions, based solely on star counts as shown in Table 1. It also lists the total number of sub-regions extracted under each criterion, along with their corresponding areas. The area of the largest sub-regions is $(8.88 \times 8.88) \operatorname{arcmin}^{2}$ and the smallest is $(2.22 \times 4.44) \operatorname{arcmin}^{2}$. There are a total of 1322 sub-regions analysed after finer area binning. Figs 4 and 5 show the plot of $N_{p}$ versus $N$, and $N_{p}$ versus $r$ after finer area binning. There are now more regions with $r>0.5$, and the value of $N_{p}$ is confined to lower and similar values for all the six binning criteria. We still have some regions with low value of $r$, which we found are due to either poorly defined or very broad RGB. As found by Subramanian \& Subramaniam (2009, 2012) the line-of-sight (LOS) depth of the SMC is almost constant within the inner SMC (our studied region). Thus, our method of RGB slope estimation is expected not to be strongly affected by LOS depth. However, there are regions with variation in depth in the central and north-east of the SMC. This variation in depth could contribute to poor RGB slope estimation in a minority of sub-regions.

To carry forward our analysis by eliminating regions with poor fit, we choose cut-off criteria on the estimated parameters $N_{p}$, $r$, and $\sigma_{\text {slope }}$ (Paper I). Figs 6 and 7 show the plot of $N_{p}$ versus |slope $\mid$ and $\sigma_{\text {slope }}$ versus $r$, respectively. In Fig. 6, regions with $N_{p}<10$ and very large value for |slope| signify a sparsely populated RGB. Hence, the large slope value may be an artefact. Thus, to exclude regions with poorly populated RGB we select only those regions that have $N_{p} \geq 10$, implying that the fitted RGB should at least have 30 stars. Fig. 7 shows that most of the regions have $r$ in the range of $0.4-0.95$, and $\sigma_{\text {slope }}$ in the range of $0.5-2.0$. By contrast, there is a large scatter observed for regions with $\sigma_{\text {slope }}>2.0$ and $r<0.4$. Also, the clumpiest part of the figure is seen for $\sigma_{\text {slope }}<1.5$ and $r>0.5$. Thus, based on Fig. 7, we consider four different cut-

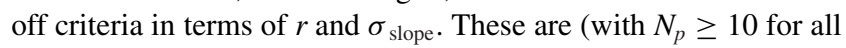
criteria) as follows:

(i) Criterion (I): $r \geq 0.4$ and $\sigma_{\text {slope }} \leq 2.0$.

(ii) Criterion (II): $r \geq 0.4$ and $\sigma_{\text {slope }} \leq 1.5$.

(iii) Criterion (III): $r \geq 0.5$ and $\sigma_{\text {slope }} \leq 2.0$.

(iv) Criterion (IV): $r \geq 0.5$ and $\sigma_{\text {slope }} \leq 1.5$.

We have plotted a histogram of the slope for all the four cut-off criteria along with the original distribution with no cut-off in Fig. 8. Comparing this with the RGB slope distribution of the LMC for OGLE III data (fig. 8 of Paper I), we find that the distributions 
(a)

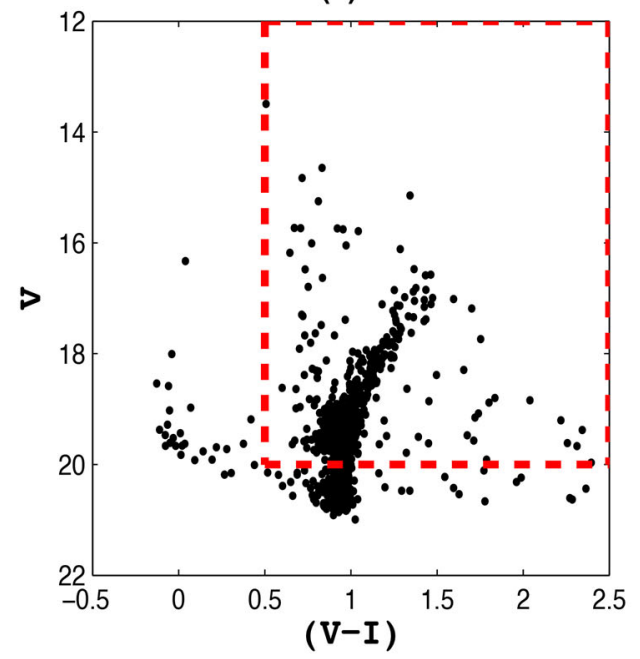

(c)

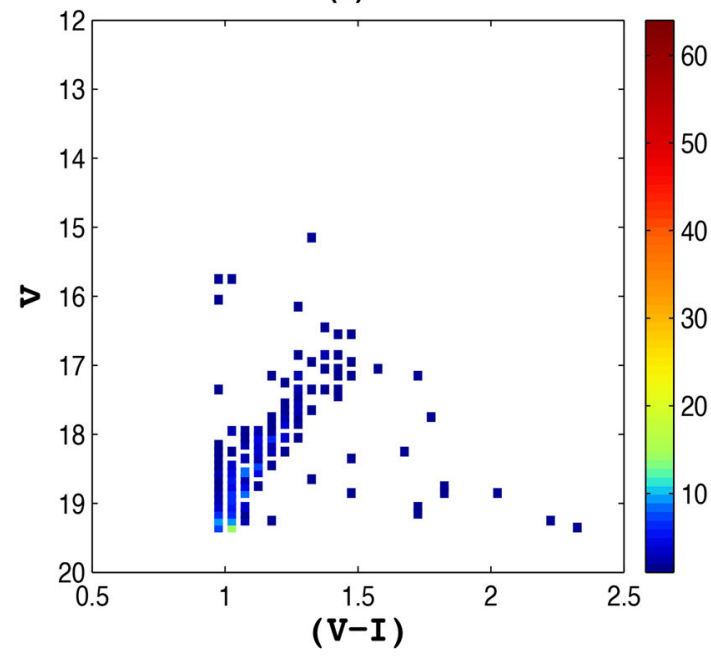

(b)

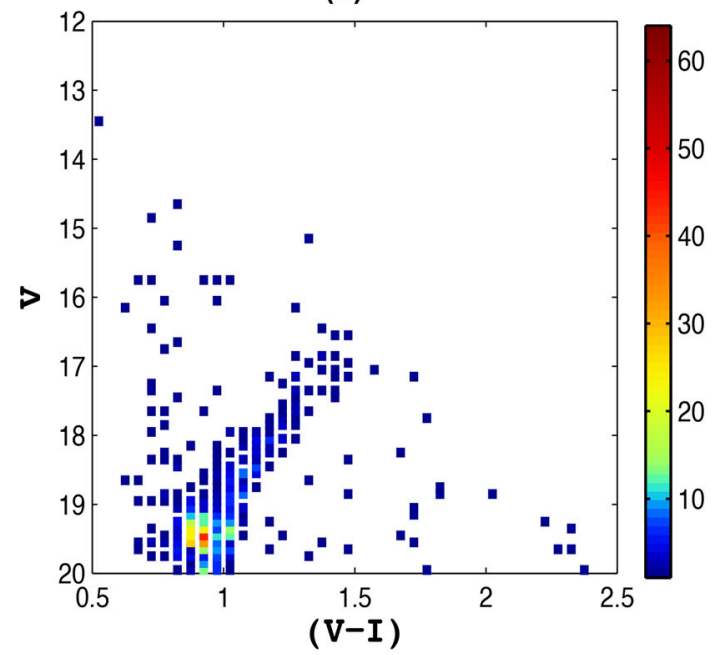

(d)

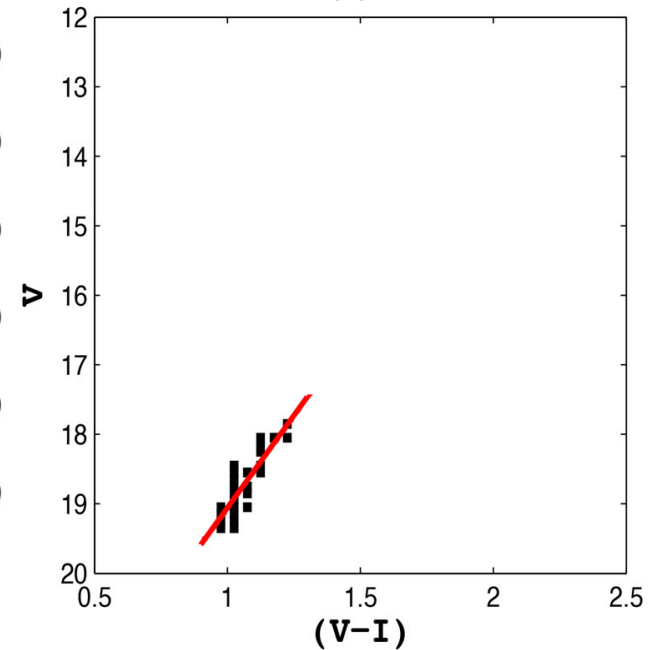

Figure 1. (a) The $V$ versus $(V-I)$ CMD of an OGLE III region at $\left(8.39^{\circ},-72.51^{\circ}\right)$, of size $(8.88 \times 8.88)$ arcmin ${ }^{2}$, with $N=952$ stars $($ black filled circles). The stars within the rectangle (red dashed line) belongs to the evolved part of the CMD. (b) Density diagram of the evolved part of CMD, where the CMD bins are colour coded based on the number of stars contained in them, as denoted in the colour bar. (c) Density diagram after giving a colour-magnitude cut at the peak value of RC distribution. (d) Density diagram showing CMD bins that have $\geq 3$ stars (black). Straight line fit to these bins representing the RGB, after $3 \sigma$ clipping, is shown as a red solid line. The estimated parameters are: $\mid$ slope $\mid=5.31 \pm 0.66, r=0.85$, and $N_{p}=26$.

appear to be different from each other in peaks and width, apart from the fact that SMC has fewer sub-regions compared to the LMC. The SMC slope distribution shows two prominent peaks, a primary at $\sim 5$ and a secondary at $\sim 4$, whereas the LMC distribution is peaked primarily at $\sim 3.5$, indicating a metal-rich case compared to the SMC. Fig. 8 shows that as the cut-off criteria become stringent from (I) to (IV), the regions with lower values of slopes $(<3)$ are mostly removed. However, the effect of increasing the cut-off for $r$ for similar values of $\sigma_{\text {slope }}$ is more than that of lowering the cutoff for $\sigma_{\text {slope }}$ for same value of $r$. This effect is similar to that we noticed for the LMC, where slope with values $<2$ were mostly removed. Thus, our last criterion (IV) is the most stringent one and the first one is the most relaxed one for selecting regions with best fits. The range of RGB slope for the SMC is found to be primarily within $\sim 3-6$ (with a few values lower than 3 and greater than 6). The LMC, on the other hand, had a different slope range, primarily varying between $\sim 2$ and 6 .

\subsection{Calibration of RGB slope to metallicity}

After estimating the RGB slope of sub-regions within the SMC, the next task is to convert the slope to metallicity. As the slope of the RGB is a measure of the average metallicity of the region, we use the spectroscopically estimated average metallicity of red giants in that region to build the required relation. We define this relation based on the spectroscopically determined mean metallicity for several regions.

We used metallicities of 3037 field red giants from Dobbie et al. (2014b) to calibrate the OGLE III RGB slope map. The reasons behind using their study are: (1) They derived metallicities for the same population as our study, the RGBs; (2) Our RGB slope has a range of values and hence requires a corresponding range in metallicity to formulate a relation between the two. To our knowledge, Dobbie et al.'s work is the most extensive spectroscopic study of SMC field RGs in terms of the number of stars and the spatial area covered. Also, their study spans a large range $(\sim-0.5$ dex to about 


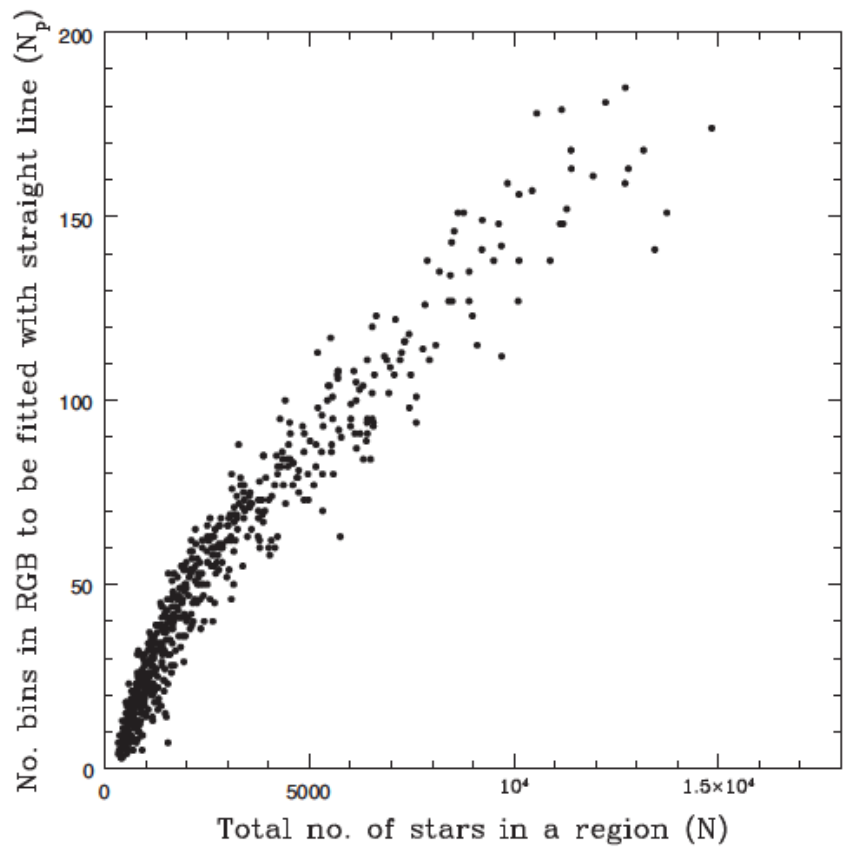

Figure 2. Plot of number of bins in RGB to be fitted with straight line $\left(N_{p}\right)$ versus the total number of stars $(N)$ for OGLE III sub-regions, after initial area binning.

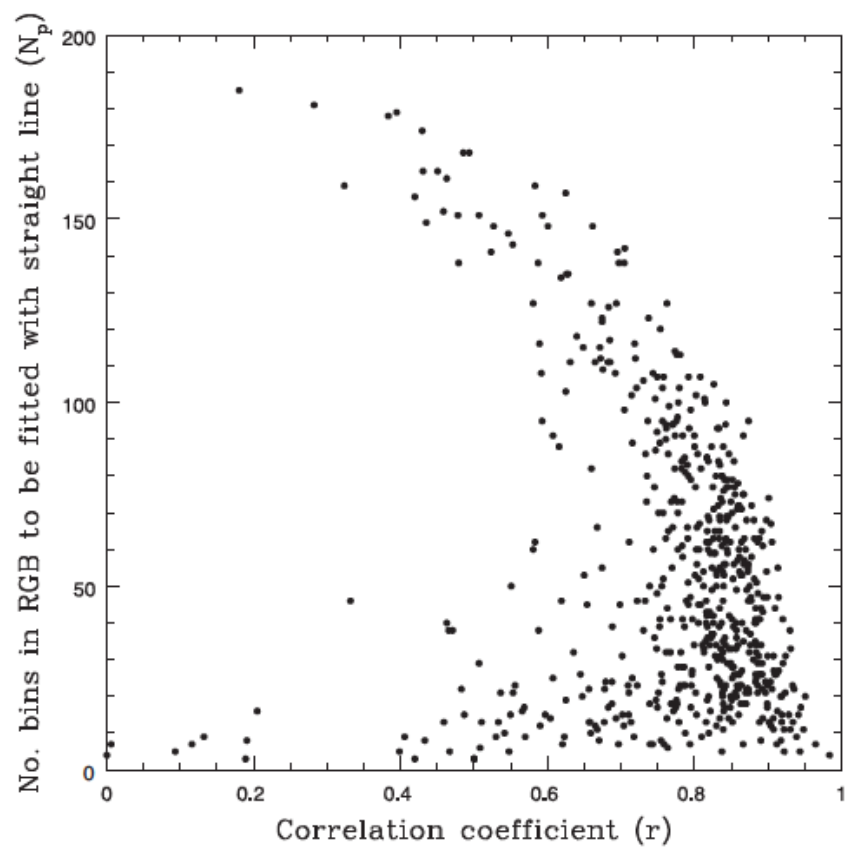

Figure 3. Plot of number of bins in RGB to be fitted with straight line $\left(N_{p}\right)$ versus correlation coefficient $(r)$ for OGLE III sub-regions, after initial area binning.

-2.5 dex) in metallicity. We calculate the mean metallicity for a sub-region by averaging over the Dobbie et al. metallicities within its area. While doing so, we consider stars lying within twice the standard deviation about the mean metallicity. To ensure good calibration we consider only those sub-regions that have $r$ higher than $0.70, \sigma_{\text {slope }}$ lower than 1.0 , and contain spectroscopic metallicity estimate of at least 5 RGs. These cut-off conditions used are similar to that used for calibrating the LMC metallicity map (section 3.1 of Paper I).

The sub-regions used for calibration are plotted in the metallicity versus $\mid$ slope $\mid$ plane. The trend shows that with increasing value of |slope|, the metallicity decreases. We estimate a linear relation between them by fitting a straight line using least-squares fit with $2 \sigma$ clipping. In Fig. 9, the final 33 sub-regions used for the slopemetallicity calibration are shown, and these values are also tabulated in Table 2. The slope-metallicity relation estimated is given by

$[\mathrm{Fe} / \mathrm{H}]=(-0.098 \pm 0.029) \times \mid$ slope $\mid+(-0.490 \pm 0.140) ;$

with $r=0.51$. The estimated slope $(-0.098)$ of this RGB slopemetallicity relation is shallower when compared to that of the LMC in Paper I $(-0.137$, their equation 1). The $y$-intercept $(-0.489)$ of the slope-metallicity relation is metal poor for the SMC as compared to the LMC (0.092) owing to the galaxy being relatively metal poor. It is to be noted that for the SMC we have been able to establish the relation with a larger number of data points (33) than for the LMC (16). Also, the range of $\mid$ slope $\mid$ for these points $(\sim 3.2 \lesssim \mid$ slope $\mid \lesssim$ 6.2) almost covers the entire slope distribution of the SMC (Fig. 8). Whereas, for the LMC the slope range of the calibrators $(3.2 \lesssim$ |slope $\mid \lesssim 4.6$ ) covered mainly the peak of the RGB slope distribution (fig. 8 of Paper I). Thus, we have successfully established a slopemetallicity relation for the SMC for OGLE III data set.

\subsection{OGLE III metallicity map}

We use equation (1) to convert all the RGB slopes to metallicity. The OGLE III metallicity maps are shown in Figs 10 and 11 (for the cut-off criteria I and IV, respectively), plotted in Cartesian coordinate system $(X, Y)$ to understand the variation of metallicity in the plane of the sky. The number of regions with poor fit (mostly with slope lower than 3 in Fig. 8) decreases as one moves from criteria I to IV. The number of such regions are only a few tens. Also, the metallicity maps using cut-off criteria II and III appear very similar and do not add much value to our results. Thus, we prefer to present only the extreme cases, one with the relaxed cutoff (I) and one with the most stringent cut-off (IV) to bring out the differences in our results. The value of the SMC centre used is $\mathrm{RA}=0^{h} 52^{m} 12.5^{s}$; Dec. $=-72^{\circ} 49^{m} 43^{s}$ (J2000.0; de Vaucouleurs \& Freeman 1972).

The metallicity trend in the central SMC, eastern and western wings are seen in the OGLE III metallicity maps. The central region is metal rich but not homogeneous. There seems to be a very shallow gradient as one goes out from the centre towards the eastern or western regions. As we move from criteria I to IV, more regions with poor fit are excluded from the map but the overall appearance hardly varies. The regions removed due to poor slope estimation are located in the centre and northeast, and are primarily the star-forming regions. Such regions suffer from problem due to differential reddening and/or multiple-dominant population. The sub-regions with variation in LOS depth in the central and northeast part of the SMC that contribute to poor RGB slope-estimation also get removed in our analysis using the cut-off criteria. In both maps, the metallicity mainly varies from -0.8 dex to about -1.1 dex, with only a very few points more metal rich than -0.8 dex (mostly located near the central region) or more metal poor than -1.1 dex (mostly located away from the central region).

Fig. 12 shows a histogram of metallicity for cut-off criteria I and IV. The distribution is binned with a width of $0.15 \mathrm{dex}$, which is of the order of one sigma error. The distribution contains smaller number of regions with high metallicity as we progressively tighten 
Table 1. Sub-division of OGLE III regions.

\begin{tabular}{lccccccc}
\hline Sl. no. & $\begin{array}{c}\text { No. of } \\
\text { stars }\end{array}$ & $\begin{array}{c}\text { No. of } \\
\text { regions } \\
(a)\end{array}$ & $\begin{array}{c}\text { No. of } \\
\text { division } \\
\text { along RA } \\
(b)\end{array}$ & $\begin{array}{c}\text { No. of } \\
\text { division } \\
\text { along Dec. } \\
(c)\end{array}$ & $\begin{array}{c}\text { No. of } \\
\text { sub-divisions } \\
(d=b \times c)\end{array}$ & $\begin{array}{c}\text { Area of } \\
\text { a sub-division } \\
\left(\operatorname{arcmin}^{2}\right)\end{array}$ & $\begin{array}{c}\text { Number of } \\
\text { sub-regions } \\
(a \times d)\end{array}$ \\
\hline 1 & $0<N \leq 1600$ & 266 & 1 & 1 & 1 & $(8.88 \times 8.88)$ & 266 (black) \\
2 & $1600<N \leq 3600$ & 160 & 2 & 1 & 2 & $(4.44 \times 8.88)$ & 320 (brown) \\
3 & $3600<N \leq 5000$ & 51 & 3 & 1 & 3 & $(2.96 \times 8.88)$ & 153 (red) \\
4 & $5000<N \leq 7200$ & 58 & 2 & 2 & 4 & $(4.44 \times 4.44)$ & 232 (orange) \\
5 & $7200<N \leq 11000$ & 39 & 3 & 2 & 6 & $(2.96 \times 4.44)$ & 234 (yellow) \\
6 & $N>11000$ & 15 & 4 & 2 & 8 & $(2.22 \times 4.44)$ & 120 (dark green) \\
\hline
\end{tabular}

Note. The table describes the six binning criteria used to sub-divide OGLE III regions. For each criterion, the second column denotes the limit on total number of stars $(N)$ within a region. The third column gives the number of regions, having $N$, within that specified limit. Columns four and five specify the number by which a region is binned along RA and Dec., respectively. Column six thus gives the total number of sub-regions, a single region is binned into. Whereas the seventh column gives the area of each such sub-region. The last (eighth) column denotes the total number of sub-regions corresponding to each of the six sub-division criteria. The colours adjacent to the numbers are used to denote them in Figs 4 and 5.

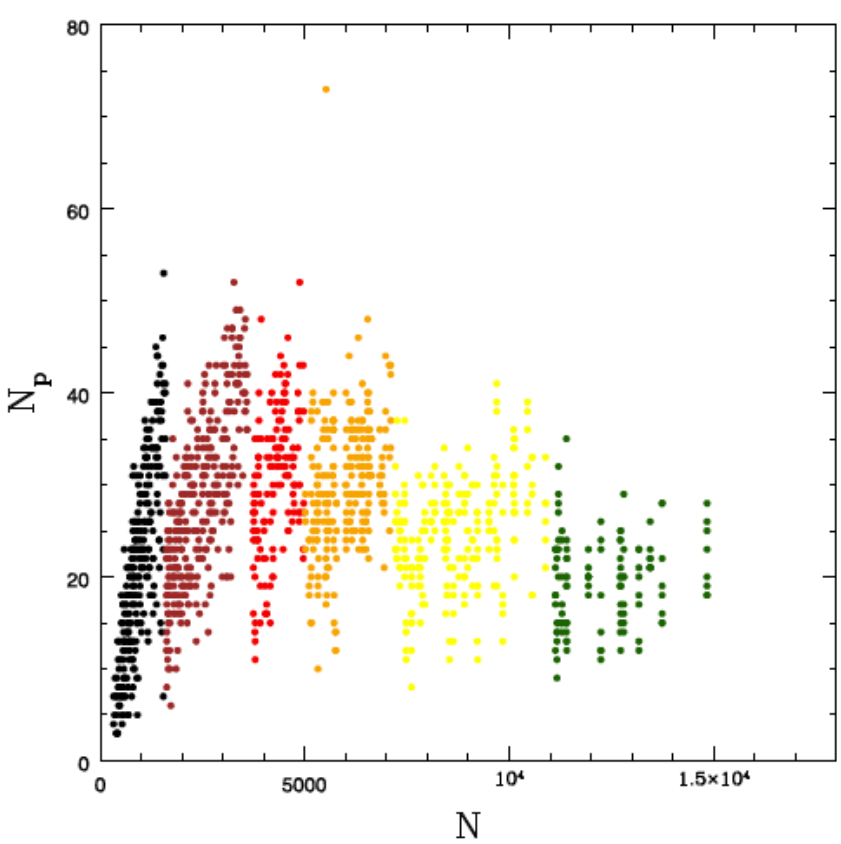

Figure 4. Plot of $N_{p}$ versus $N$ for OGLE III sub-regions, after finer area binning. The colours correspond to the six different bin areas, as mentioned in the eighth column of Table 1.

the selection criteria, from (I) to (IV). The distribution peaks at about -0.95 dex, with a secondary peak at about -0.85 dex. There are hardly any regions with metallicity higher than -0.75 dex or lower than -1.2 dex. Table 3 gives the list of mean metallicities of the SMC by region (along with their respective standard deviations) estimated for cut-off criteria I and IV. The errors mentioned alongside the mean values are the standard deviation of the average, and do not include the error in metallicity estimation of each region. The mean values estimated by criteria I and IV are similar, though the number of regions considered changes. Nevertheless, criterion IV being the most stringent one gives us the mean metallicity of the SMC. However, this value of metallicity estimate excludes the northern and southern parts of the SMC. To analyse those areas, we use MCPS data, discussed in the following section.

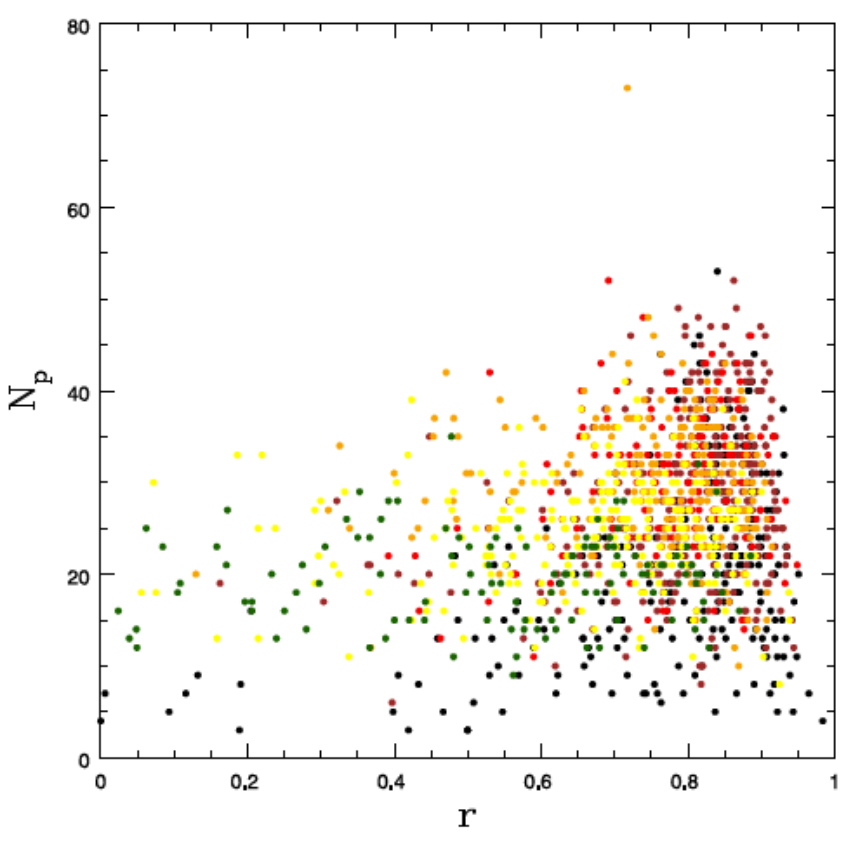

Figure 5. Plot of $N_{p}$ versus $r$ for OGLEIII sub-regions, after finer area binning. The colours correspond to the six different bin areas, as mentioned in the eighth column of Table 1.

\section{MCPS ANALYSIS}

A similar analysis was carried out for the MCPS data, where the observed region is binned into 778 small sub-regions, each of dimension $(8.9 \times 10.0) \operatorname{arcmin}^{2}$ in RA and Dec. As already mentioned in Section 2, this survey covers a larger area than the OGLE III survey. Although its resolution is comparatively lower than the OGLE III survey, the MCPS provides more coverage on the northern and southern regions of the central SMC. We adopt the same procedure to estimate the slope of the RGB as was done for OGLE III analysis, starting from isolating the RGB, locating the densest point of the $\mathrm{RC}$, and estimating the density distribution of stars on the RGB, and then estimating the RGB slope. The denser sub-regions were further sub-divided based solely on stellar density. We adopted five binning criteria based on stellar density for the MCPS observed region as shown in Table 4. 


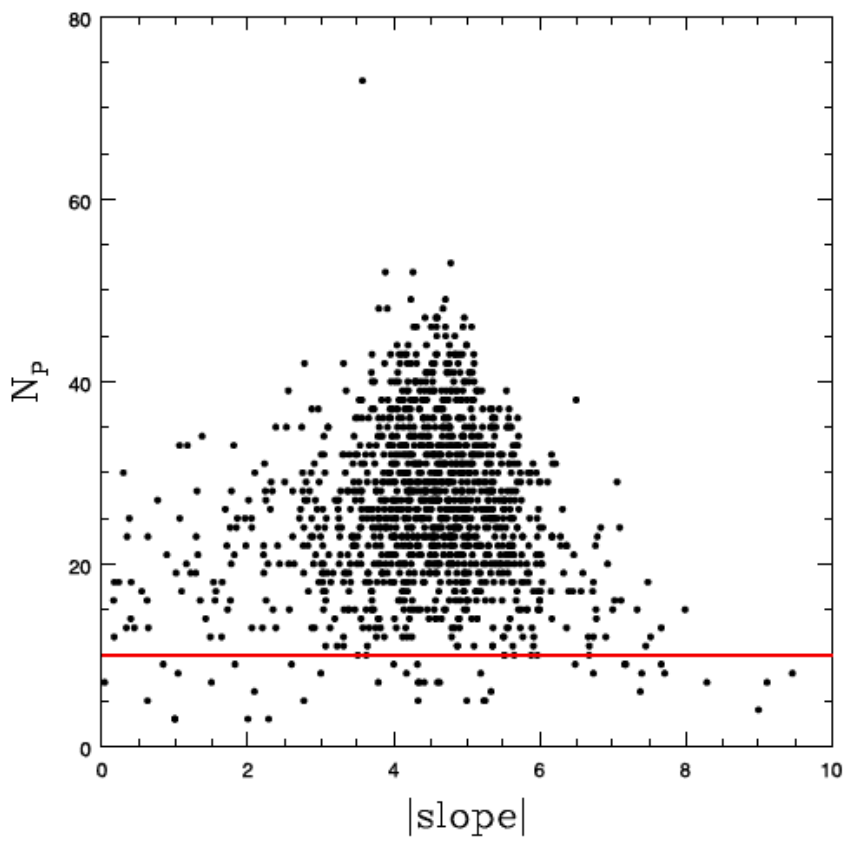

Figure 6. Plot of $N_{p}$ versus |slope| for OGLE III sub-regions. The red line at $N_{p}=10$ denotes the cut-off decided to exclude regions with poorly populated RGB.

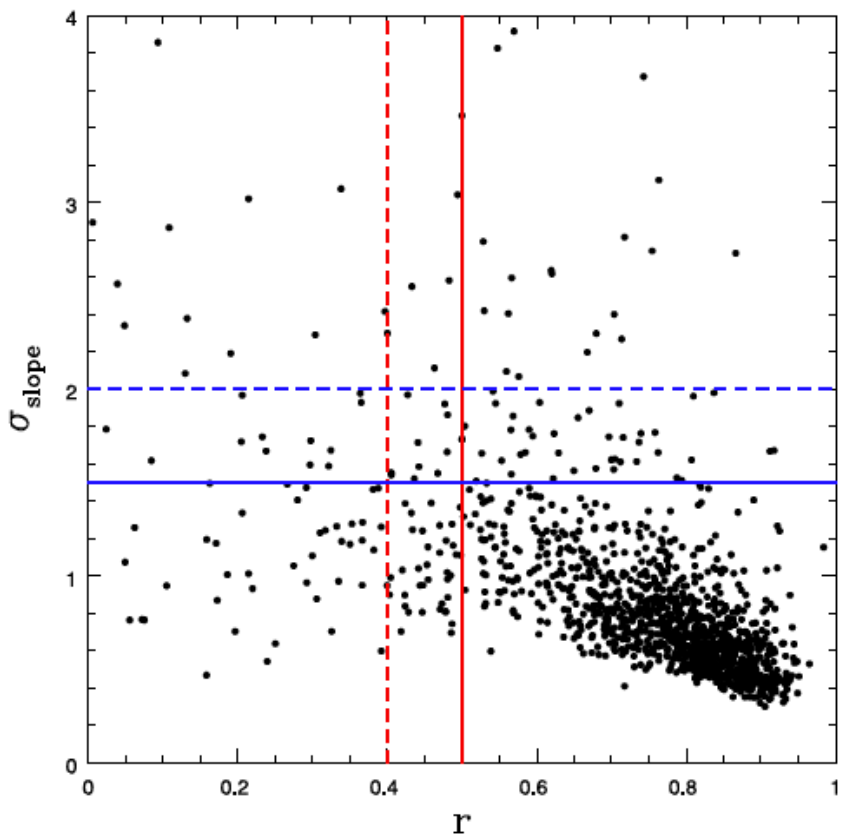

Figure 7. Plot of $\sigma_{\text {slope }}$ versus $r$ for OGLE III sub-regions. The blue dashed and solid lines correspond to the cut-off criteria on $\sigma_{\text {slope }}$ at 2.0 and 1.5, respectively. The red dashed and solid lines denote the cut-off corresponding to $r$ at 0.4 and 0.5 , respectively.

There are 1356 sub-regions analysed after finer area binning for which the parameters are estimated. The largest bins are $(8.90 \mathrm{arcmin} \times 10.0 \mathrm{armin})$, and the smallest bins are (2.97 arcmin $\times 5.0$ arcmin). Figs 13 and 14 show the plot of $N_{p}$ versus $N$ and $N_{p}$ versus $r$, respectively, before finer area binning. For comparison, Figs 15 and 16 show the plot of $N_{p}$ versus $N$ and $N_{p}$ versus $r$, respectively, after finer area binning. Similar to the OGLE III analysis, while finely binning the observed area spatially, we have

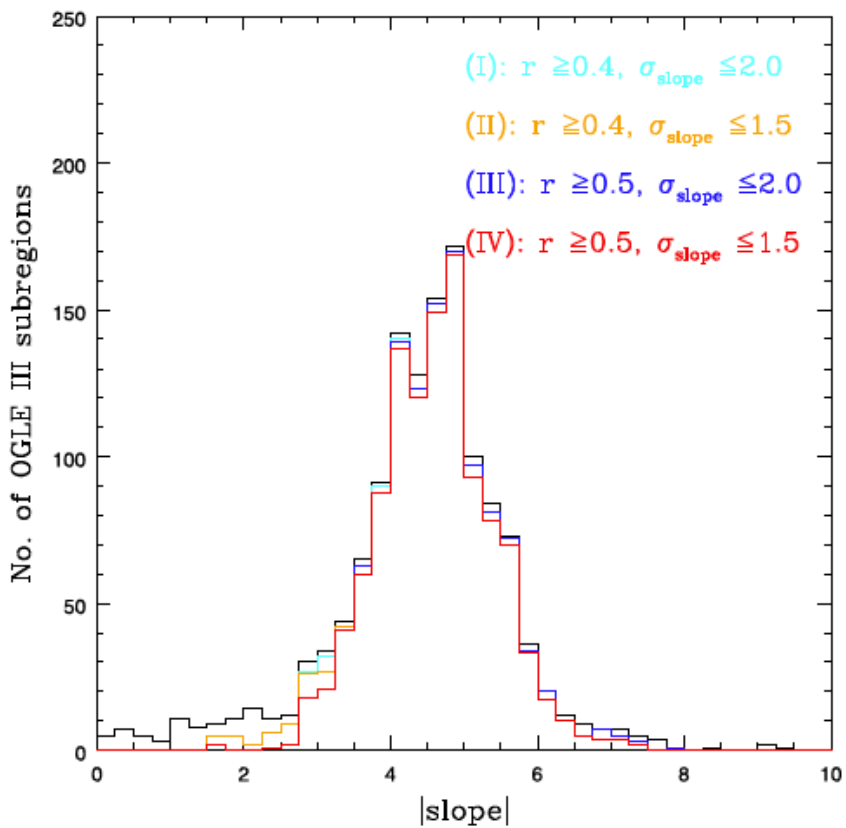

Figure 8. Histogram of |slope| for OGLE III sub-regions estimated for all the four cut-off criteria [(I) in cyan, (II) in orange, (III) in blue, and (IV) in red]. $N_{p} \geq 10$ for all these four cases. The black solid line shows the distribution of |slope| with no cut-off corresponding to all OGLE III sub-regions.

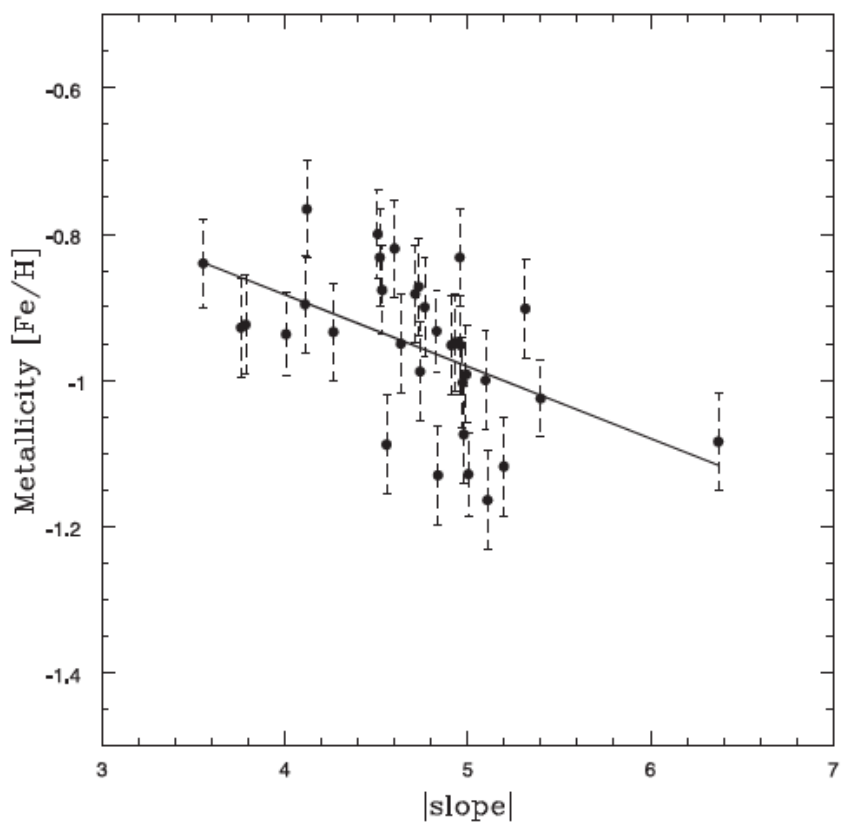

Figure 9. Plot of $[\mathrm{Fe} / \mathrm{H}]$ versus |slope| for OGLE III data. The points denote our sub-regions whose mean $[\mathrm{Fe} / \mathrm{H}]$ has been found using RGs from Dobbie et al. (2014b), with the solid line denoting a linear relation between them. The error bar (dashed line) shown for each point is the standard error of mean $[\mathrm{Fe} / \mathrm{H}]$.

tried to constrain $N_{p}$ to similar values for all sub-regions in order to attain higher $r(\geq 0.5)$. The regions with lower values of $r(<0.5)$ are likely to be those that suffer either from issues of multiple dominant population, small-scale variation in reddening, and/or variation in LOS depth. 
Table 2. Calibrators for OGLE III slope-metallicity relation.

\begin{tabular}{|c|c|c|c|c|c|c|}
\hline $\mathrm{RA}\left({ }^{\circ}\right)$ & Dec. $\left({ }^{\circ}\right)$ & |slope| & $\sigma_{\text {slope }}$ & $r$ & $\begin{array}{c}\text { Mean }[\mathrm{Fe} / \mathrm{H}] \\
(\mathrm{dex})\end{array}$ & $\begin{array}{l}\text { Standard error } \\
\text { of mean }[\mathrm{Fe} / \mathrm{H}]\end{array}$ \\
\hline 11.82 & -71.88 & 4.93 & 0.32 & 0.93 & -0.95 & 0.07 \\
\hline 13.35 & -74.55 & 5.00 & 0.50 & 0.86 & -1.13 & 0.06 \\
\hline 14.57 & -71.49 & 4.53 & 0.39 & 0.88 & -0.88 & 0.06 \\
\hline 14.08 & -71.50 & 5.11 & 0.81 & 0.75 & -1.16 & 0.07 \\
\hline 18.88 & -72.38 & 4.91 & 0.57 & 0.86 & -0.95 & 0.07 \\
\hline 18.39 & -72.23 & 5.40 & 0.61 & 0.84 & -1.02 & 0.05 \\
\hline 18.86 & -72.23 & 4.98 & 0.55 & 0.88 & -1.07 & 0.07 \\
\hline 18.87 & -72.07 & 6.37 & 0.67 & 0.90 & -1.08 & 0.07 \\
\hline 8.90 & -72.50 & 4.97 & 0.50 & 0.87 & -1.00 & 0.06 \\
\hline 8.13 & -74.15 & 4.84 & 0.54 & 0.81 & -1.13 & 0.07 \\
\hline 13.13 & -71.88 & 4.74 & 0.47 & 0.89 & -0.99 & 0.07 \\
\hline 11.92 & -72.19 & 4.73 & 0.39 & 0.89 & -0.87 & 0.07 \\
\hline 13.53 & -71.93 & 4.71 & 0.38 & 0.91 & -0.88 & 0.07 \\
\hline 13.77 & -71.93 & 4.96 & 0.49 & 0.85 & -0.95 & 0.07 \\
\hline 14.91 & -71.64 & 4.83 & 0.79 & 0.84 & -0.93 & 0.06 \\
\hline 13.72 & -71.80 & 3.78 & 0.78 & 0.71 & -0.92 & 0.07 \\
\hline 13.96 & -71.80 & 4.11 & 0.56 & 0.80 & -0.90 & 0.07 \\
\hline 14.19 & -71.80 & 4.96 & 0.70 & 0.80 & -0.83 & 0.07 \\
\hline 17.10 & -72.08 & 4.63 & 0.63 & 0.87 & -0.95 & 0.07 \\
\hline 17.47 & -72.52 & 5.20 & 0.69 & 0.81 & -1.12 & 0.07 \\
\hline 11.35 & -74.28 & 4.99 & 0.52 & 0.89 & -0.99 & 0.07 \\
\hline 16.66 & -72.82 & 4.51 & 0.59 & 0.84 & -0.80 & 0.06 \\
\hline 16.66 & -72.67 & 4.52 & 0.54 & 0.86 & -0.83 & 0.07 \\
\hline 16.82 & -72.67 & 3.55 & 0.51 & 0.80 & -0.84 & 0.06 \\
\hline 16.99 & -72.67 & 5.10 & 0.81 & 0.81 & -1.00 & 0.07 \\
\hline 16.66 & -72.52 & 3.76 & 0.67 & 0.71 & -0.93 & 0.07 \\
\hline 13.14 & -72.15 & 5.31 & 0.60 & 0.88 & -0.90 & 0.07 \\
\hline 16.27 & -72.71 & 4.26 & 0.65 & 0.79 & -0.93 & 0.07 \\
\hline 10.13 & -73.14 & 4.55 & 0.54 & 0.81 & -1.09 & 0.07 \\
\hline 10.38 & -73.14 & 4.76 & 0.64 & 0.80 & -0.90 & 0.07 \\
\hline 10.46 & -72.92 & 4.12 & 0.57 & 0.76 & -0.77 & 0.07 \\
\hline 12.79 & -73.54 & 4.01 & 0.62 & 0.79 & -0.94 & 0.06 \\
\hline 11.35 & -72.77 & 4.60 & 0.95 & 0.75 & -0.82 & 0.07 \\
\hline
\end{tabular}

Note: The table lists out the 33 calibrators used to construct the slope-metallicity relation for OGLE III data. The central (RA, Dec.) corresponding to each calibrator is listed down in first and second column, respectively. The third and fourth columns give the estimated slope and its associated error, respectively, whereas the correlation coefficient for each case is specified in the fifth column. The sixth column denotes the mean $[\mathrm{Fe} / \mathrm{H}]$ estimated for each calibrator using spectroscopic results of Dobbie et al. (2014b). The standard error of mean $[\mathrm{Fe} / \mathrm{H}]$ is mentioned in the last column. It is calculated as $0.15 / \sqrt{n}$, where $n$ is the number of spectroscopically studied RGs located within the area of the sub-region.

Similar to OGLE III analysis (Section 3), we use four cut-off criteria shown in Fig. $17\left(N_{p}\right.$ and |slope|) and Fig. 18 ( $\sigma_{\text {slope }}$ versus $r)$ to filter out regions with poor slope estimations. The cut-off values for $r$ and $\sigma_{\text {slope }}$ (with $N_{p} \geq 10$ for all cases) are same as that were used for OGLE III. The slope distribution for all the four cut-off criteria are shown in Fig. 19, along with the distribution with no cut-offs. The figure shows that, as the $r$ cut-off becomes more stringent, the width of the histogram decreases such that the lower slope values are removed. The effect of $\sigma_{\text {slope }}$ is insignificant on the distribution for same cut-off in $r$. The strictest cut-off, $N_{p} \geq 10, r \geq$ 0.5 , and $\sigma_{\text {slope }} \leq 1.5$ shows that slope values ranges primarily from 2 to 6 for the regions studied here.

Although both OGLE III and MCPS deal with same optical bands ( $V$ and $I$ filters), there may be systematic differences between their filter systems. In Paper I (their equation 2), we found that there exists a systematic difference between their $I$ bands (not in the $V$ band). Therefore, we cannot directly compare the RGB slope distributions estimated from these two data sets. Thus, it is required to establish an independent slope-metallicity relation for the
MCPS data, so as to bring the two surveys on to the same scale in metallicity.

\subsection{Calibration of RGB slope to metallicity}

We adopt similar technique as in Section 3.1 for OGLE III case to estimate a slope-metallicity relation for MCPS data set. Since MCPS has a larger spatial coverage than OGLE III, we are able to employ more data points to calibrate the RGB slopes. The metallicity and slope ranges obtained for the MCPS calibration points are similar to that for OGLE III data set. The slope range of the calibration points also more or less covers the entire slope distribution (Fig. 19) of the MCPS data. Fig. 20 shows the MCPS slope metallicity relation derived by a linear fit after $2 \sigma$ clipping. The final calibration points (41) are listed down in Table 5.

$[\mathrm{Fe} / \mathrm{H}]=(-0.083 \pm 0.024) \times \mid$ slope $\mid+(-0.614 \pm 0.106) ;$

with $r=0.49$. This is different from the calibration of MCPS metallicity map for the LMC (section 4.2 in Paper I). There, due 


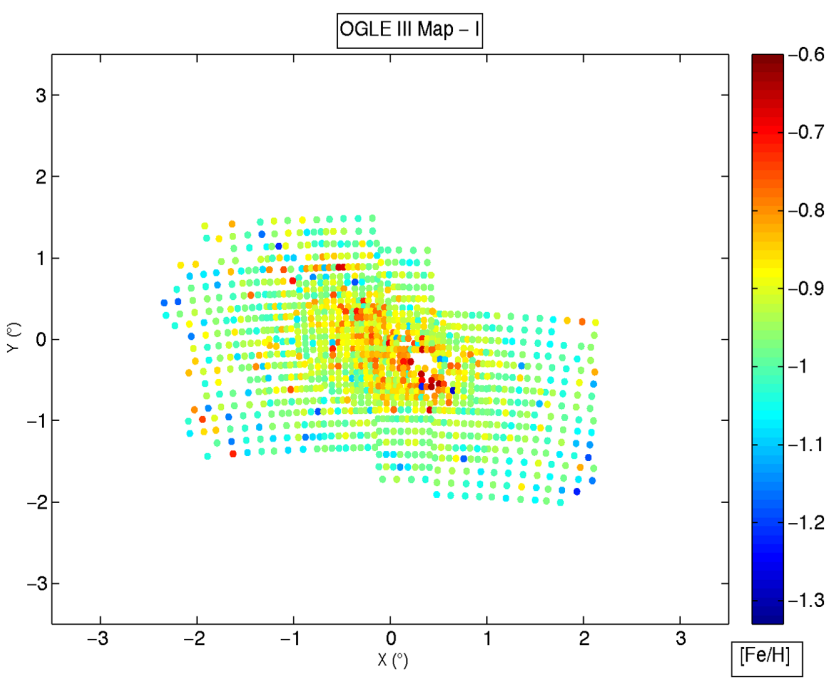

Figure 10. OGLE III metallicity map with cut-off criterion (I): $N_{p} \geq 10$, $r \geq 0.4$, and $\sigma_{\text {slope }} \leq 2.0$.

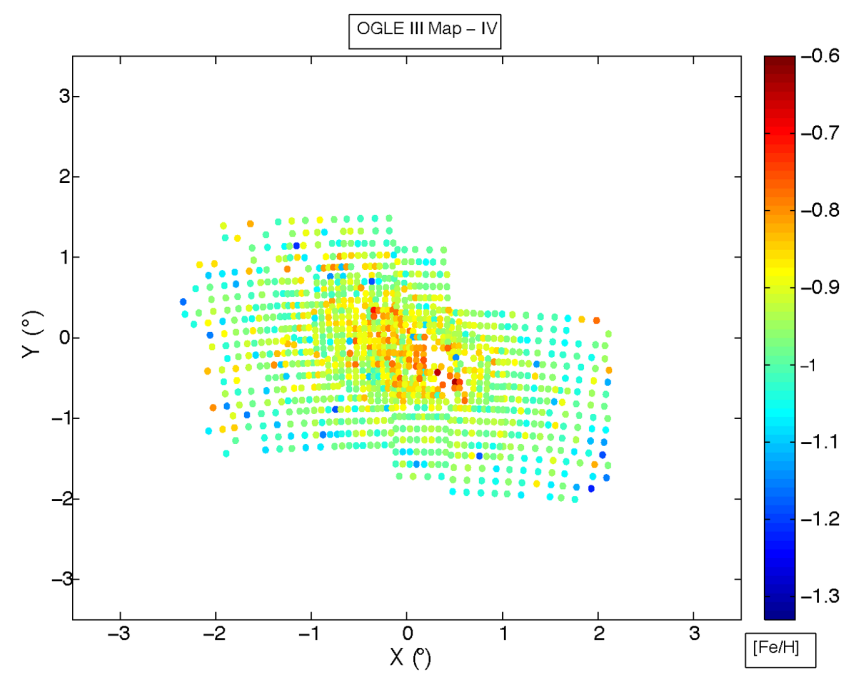

Figure 11. OGLE III metallicity map with cut-off criterion (IV): $N_{p} \geq 10$, $r \geq 0.5$, and $\sigma_{\text {slope }} \leq 1.5$.

to inadequate number of calibration points for the MCPS case, we transformed the MCPS filter system to the OGLE III filter system by correcting for their systematic difference between I filters. Then, we used the OGLE III slope metallicity relation to calibrate the MCPS slopes to metallicities. However, in this case, we successfully established an independent slope-metallicity relation for MCPS data. Thus, the OGLE III and MCPS system are now tied down to the same scale in metallicity.

\subsection{MCPS metallicity map}

The MCPS metallicity maps are shown in Figs 21 and 22 for the cut-off criteria I and IV, respectively (using equation 2), in Cartesian coordinate $(X, Y)$ system on the projected sky plane. For reasons similar to the OGLE III case, we have ignored the maps using criteria II and III and presented only the two extreme cases. The metallicity trends in the northern and southern SMC are now revealed. A qualitative comparison shows that these regions have less metallicity variations as compared to the eastern and western SMC.

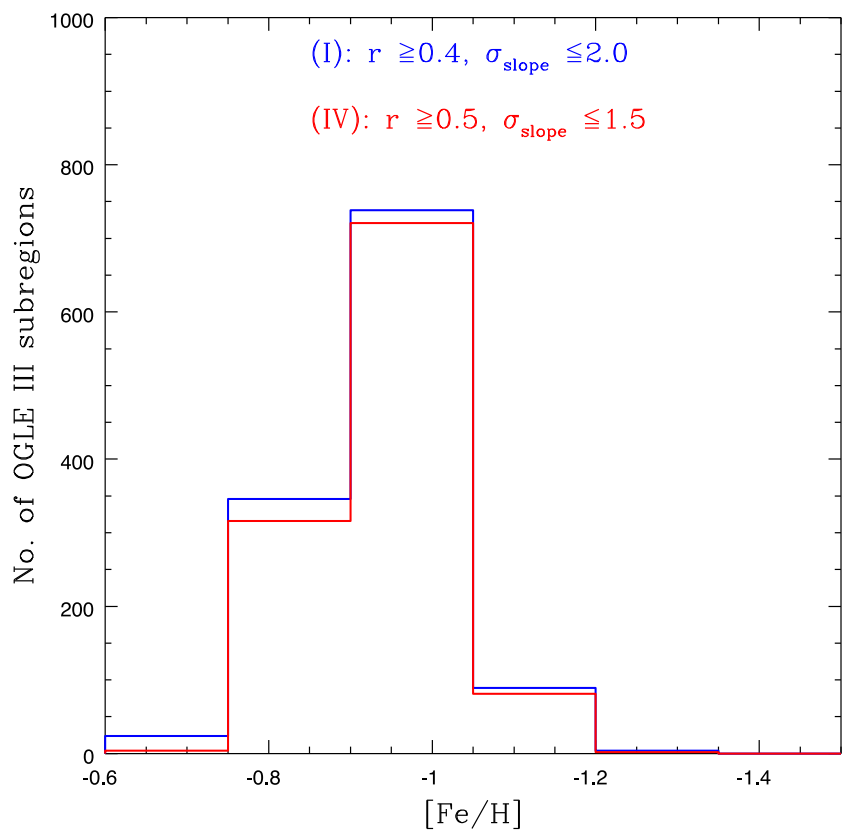

Figure 12. Histogram of $[\mathrm{Fe} / \mathrm{H}]$ for OGLE III data, estimated for cut-off criteria (I) in blue and (IV) in red. $N_{p} \geq 10$ for both cases.

The map shows a shallow gradient from central to outer SMC within $2.5 \mathrm{deg}$. Again, more regions with poor fit are excluded as we move from cut-off criteria I to V. The metallicity ranges from $-0.8 \mathrm{dex}$ to about $-1.1 \mathrm{dex}$, with only very few points more metal rich than -0.8 dex (mostly located near the central region) or more metal poor than -1.1 dex (mostly located away from the central region). The gaps in the map are due to regions with poorly estimated slopes. The regions that get missed out due to this effect are those located near the central, north-east, and star-forming regions (similar to OGLE III).

Fig. 23 shows a histogram of metallicity for cut-off criteria I and IV. The distribution is binned with a width of 0.15 dex. The distribution looks very similar to that of the OGLE III distribution (but with fewer sub-regions) and peaks at about -0.95 dex alongside a secondary peak at about -0.85 dex. Table 6 lists the mean metallicity (along with standard deviation) of the SMC estimated for cut-off criteria I and IV using the MCPS data. The errors mentioned alongside the mean values are the standard deviation of the average and does not include the error in metallicity estimation of each region. It is to be noted that the mean values for both criteria are almost similar, though the number of regions changes. The mean values also correspond to that estimated using the OGLE III data in Table 3. Since, IV is the most stringent criterion, the rest of our analysis for both data sets will be based on that.

\section{ERROR ANALYSIS}

We describe the error estimation of slope and metallicity for both data sets in this section. The method is similar to that described in Paper I (Section 5). The factors that can contribute to the error in slope estimation are the photometric error associated with individual points in the CMD (errors in $V$ and $I$ magnitudes are $\leq 0.15$ ), and error due to fine binning of the CMD (dimension of each bin is 0.05 in colour and 0.10 in magnitude). In this study, we are interested in the mean metallicity of a region, of which the RGB slope is an indicator. The above factors could contribute to the errors if we were 
Table 3. Mean metallicity for SMC using OGLE III data.

\begin{tabular}{lccccc}
\hline Cut-off criteria & $r$ & $\sigma_{\text {slope }}$ & Region of the SMC & Number of sub-regions & Mean [Fe/H] (dex) \\
\hline I & $\geq 0.40$ & $\leq 2.0$ & COMPLETE & 1201 & $-0.94 \pm 0.09$ \\
IV & $\geq 0.50$ & $\leq 1.5$ & COMPLETE & 1124 & $-0.94 \pm 0.08$ \\
\hline
\end{tabular}

Note. The first column denotes the first and fourth cut-off criteria considered to filter out the SMC sub-regions. It is to be noted that we considered $N_{p} \geq 10$ for both these cases. The second and third columns specify the constraint on correlation coefficient $(r)$ and $\sigma_{\text {slope, }}$ respectively, corresponding to each cut-off criterion. The fourth column denotes complete coverage for OGLE III data. The number of sub-regions that satisfy the cut-off criteria is mentioned in the fifth column. The mean metallicity and standard deviation for each cases are mentioned in the last (sixth) column.

Table 4. Sub-division of MPCS regions.

\begin{tabular}{lccccccc}
\hline Sl. no. & $\begin{array}{c}\text { No. of } \\
\text { stars }\end{array}$ & $\begin{array}{c}\text { No. of } \\
\text { regions } \\
(a)\end{array}$ & $\begin{array}{c}\text { No. of } \\
\text { division } \\
\text { along RA } \\
(b)\end{array}$ & $\begin{array}{c}\text { No. of } \\
\text { division } \\
\text { along Dec. } \\
(c)\end{array}$ & $\begin{array}{c}\text { No. of } \\
\text { sub-divisions } \\
(d=b \times c)\end{array}$ & $\begin{array}{c}\text { Area of } \\
\text { a sub-division } \\
\left(\operatorname{arcmin}^{2}\right)\end{array}$ & $\begin{array}{c}\text { Number of } \\
\text { sub-regions } \\
(a \times d)\end{array}$ \\
\hline 1 & $0<N \leq 2200$ & 384 & 1 & 1 & 1 & $(8.90 \times 10.0)$ & 384 (black) \\
2 & $2200<N \leq 4200$ & 148 & 2 & 1 & 2 & $(4.45 \times 10.0)$ & 294 (brown) \\
3 & $4200<N \leq 6000$ & 62 & 3 & 1 & 3 & $(2.97 \times 10.0)$ & 186 (red) \\
4 & $6000<N \leq 8000$ & 54 & 2 & 2 & 4 & $(4.45 \times 5.0)$ & 216 (orange) \\
5 & $N>8000$ & 46 & 3 & 2 & 6 & $(2.97 \times 5.0)$ & 276 (yellow) \\
\hline
\end{tabular}

Note. The table describes the five binning criteria used to sub-divide MCPS regions. The column descriptions are same as Table 1 . The colours adjacent to the numbers in the last column are used to denote them in Figs 15 and 16.

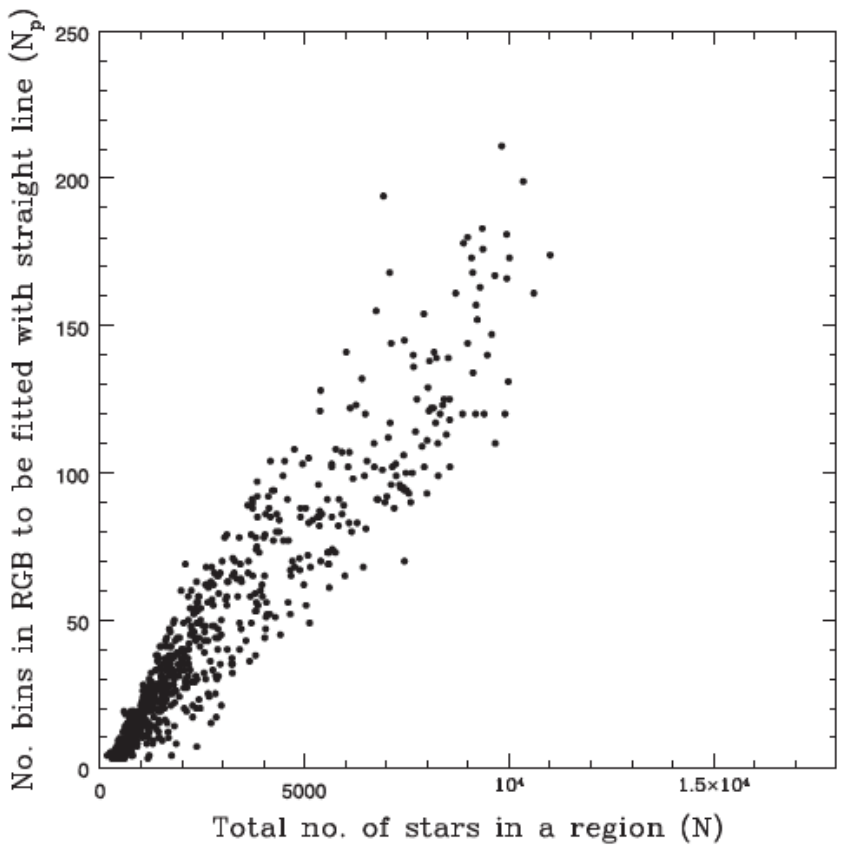

Figure 13. Plot of number of bins in RGB to be fitted with straight line $\left(N_{p}\right)$ versus the total number of stars $(N)$ for MCPS sub-regions, after initial area binning.

concerned with the probability of a star lying within a particular CMD bin and the resulting density of stars within individual CMD bins while estimating the RGB slope. However, under the present scenario we are concentrating only on the overall RGB feature and not looking into the strength of individual CMD bins. After we identify the most populated bins as a part of the RGB, the spread in the distribution of RGB bins is such that it more or less makes a good representation of the RGB. We fit these bins with the standard least-squares fitting technique to estimate the slope (|slope|) and

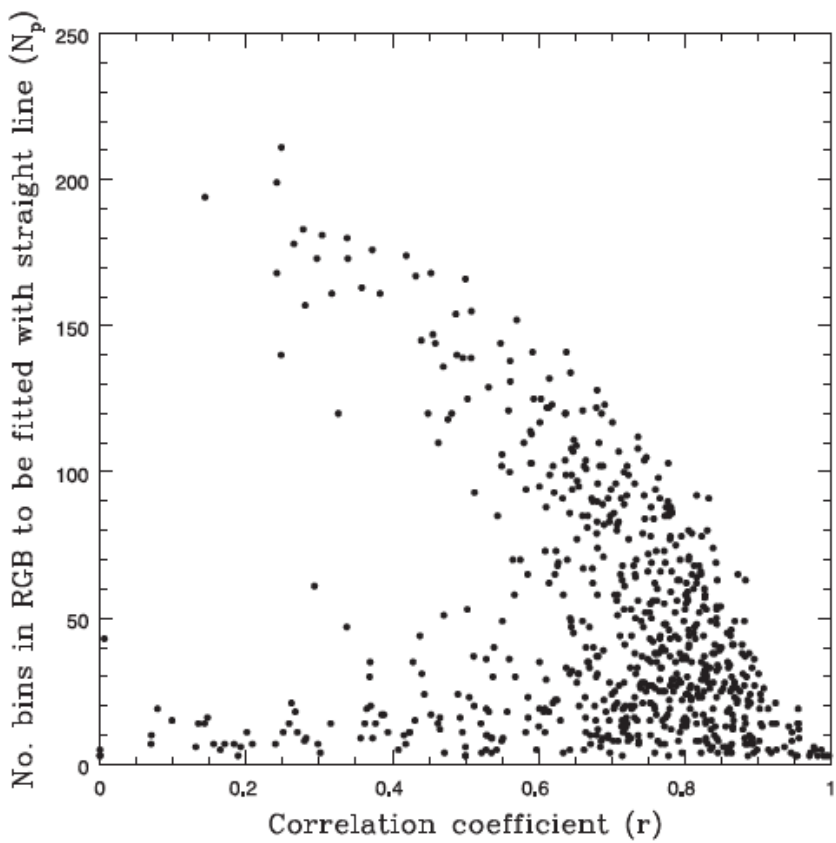

Figure 14. Plot of number of bins in RGB to be fitted with straight line $\left(N_{p}\right)$ versus correlation coefficient $(r)$ for MCPS sub-regions, after initial area binning.

its corresponding error ( $\left.\sigma_{\text {slope }}\right)$. The values of $\sigma_{\text {slope }}$ estimated in our study are relatively larger, caused by the natural spread of the populated RGB bins. This is shown in Figs 7 and 18 for OGLE III and MCPS, respectively. There we can see that for most of the regions, $\sigma_{\text {slope }}$ shows a clumpy distribution below 2 . Thus, in this study, we do not take into account the errors associated with individual stars and colour-magnitude binning when estimating the error in slope. We expect these contributions to be negligible. 


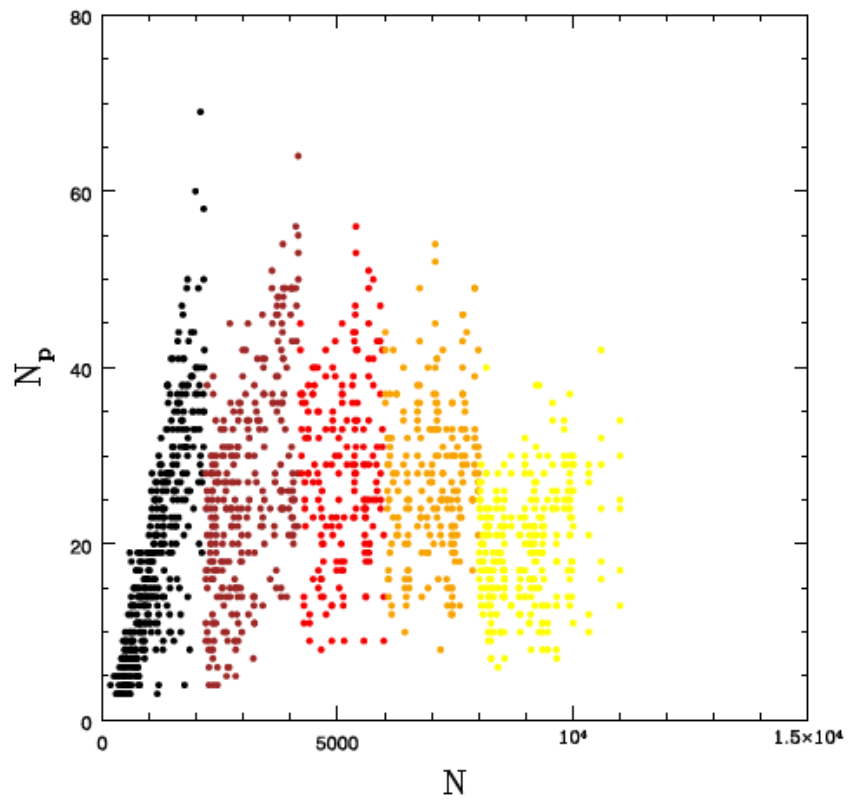

Figure 15. Plot of $N_{p}$ versus $N$ for MCPS sub-regions, after finer area binning. The colours correspond to the five different bin areas, as mentioned in the eighth column of Table 4.

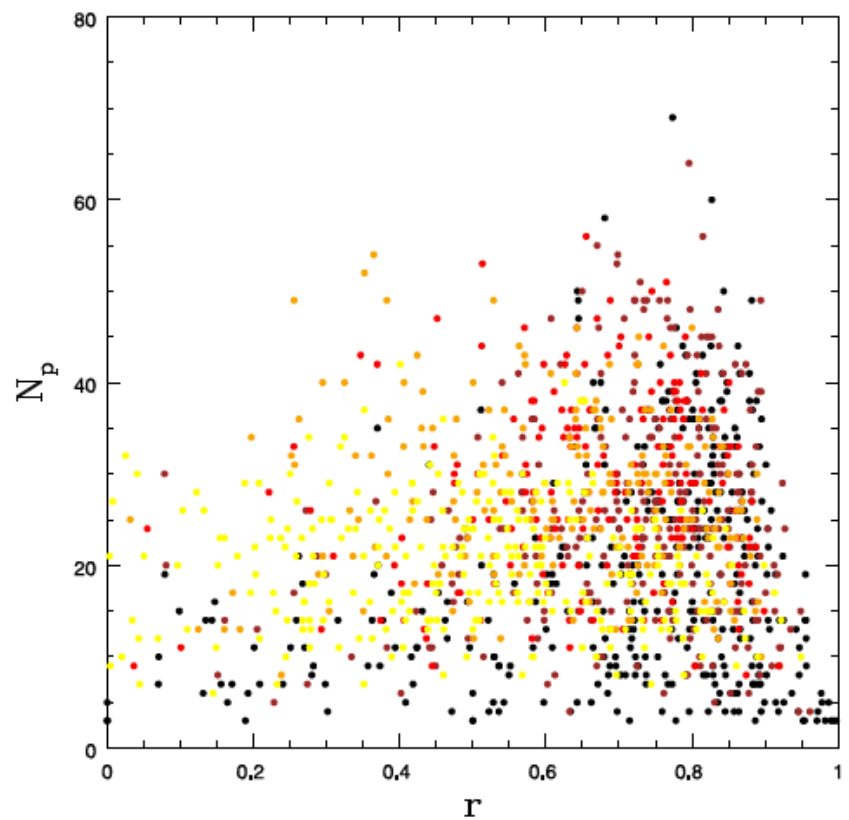

Figure 16. Plot of $N_{p}$ versus $r$ for MCPS sub-regions, after finer area binning. The colours correspond to the five different bin areas, as mentioned in the eighth column of Table 4.

The error in metallicity for individual sub-regions are calculated by propagation of errors applied to the slope-metallicity relation (equations 1 and 2) for OGLE III and MCPS individually. We can express the error associated with metallicity $\left(\right.$ error $_{[\mathrm{Fe} / \mathrm{H}]}$ ) for each sub-region as

$\operatorname{error}_{[\mathrm{Fe} / \mathrm{H}]}=\sqrt{(b \times \mid \text { slope } \mid)^{2} \times\left(\left(\frac{\sigma_{b}}{b}\right)^{2}+\left(\frac{\sigma_{\text {slope }}}{\mid \text { slope } \mid}\right)^{2}\right)+\sigma_{a}^{2}}$

where $b$ is the slope and $a$ is the $y$-intercept of the slope-metallicity relation, and $\sigma_{b}$ and $\sigma_{a}$ are the their respective errors. It is to be

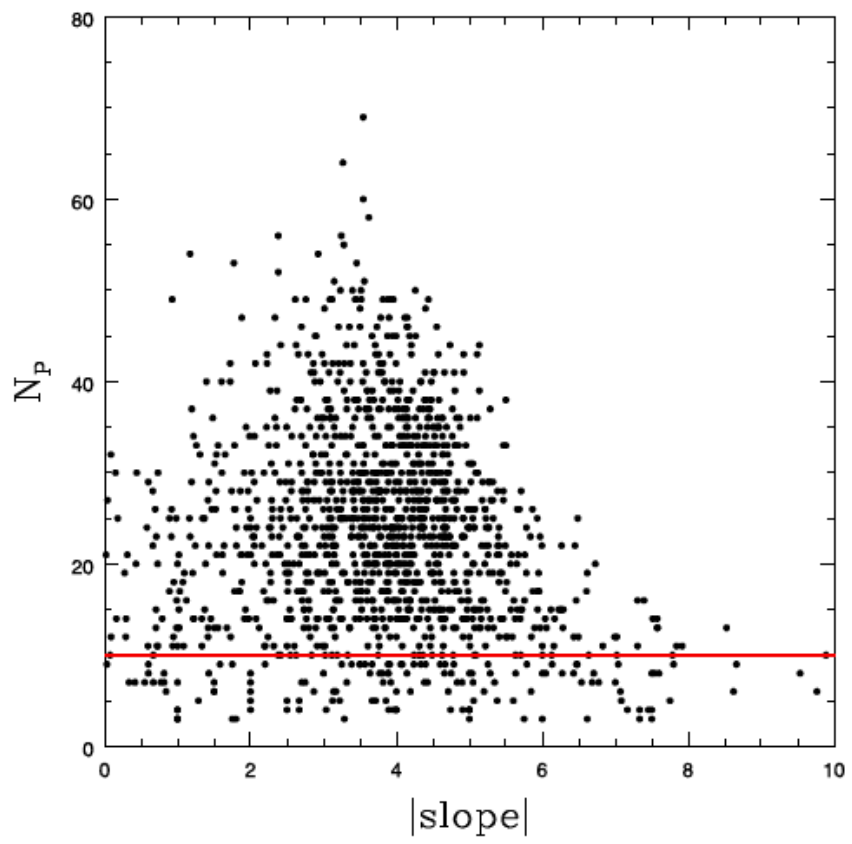

Figure 17. Plot of $N_{p}$ versus |slope| for MCPS sub-regions. The red line at $N_{p}=10$ denotes the cut-off decided to exclude regions with poorly populated RGB.

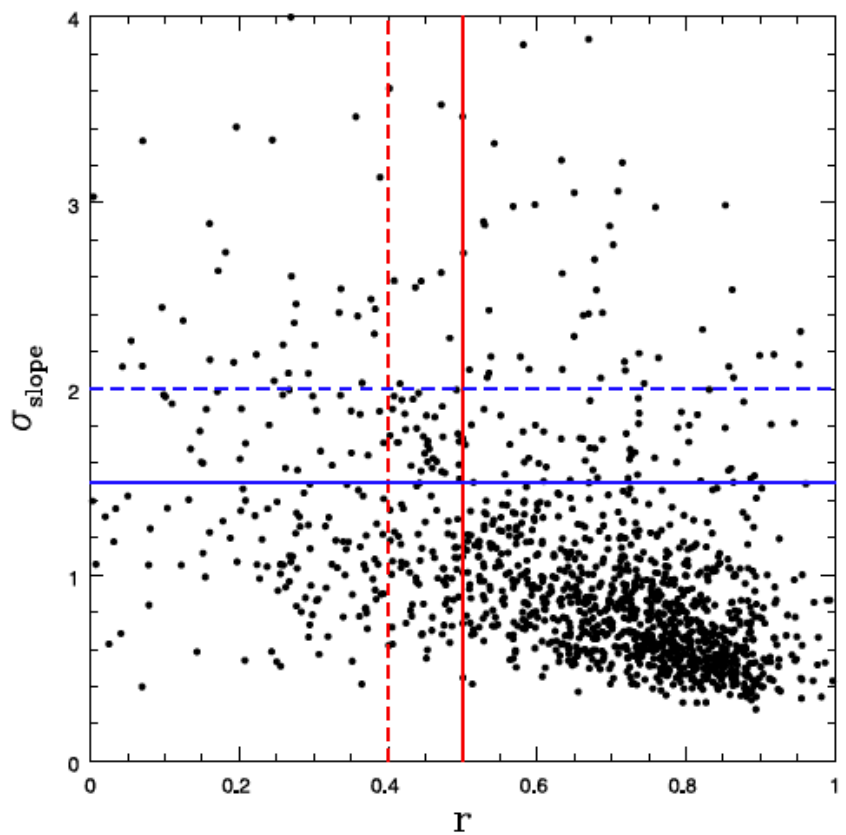

Figure 18. Plot of $\sigma_{\text {slope }}$ versus $r$ for MCPS sub-regions. The blue dashed and solid lines corresponds to the cut-off criteria on $\sigma_{\text {slope }}$ at 2.0 and 1.5, respectively. The red dashed and solid lines denote the cut-off corresponding to $r$ at 0.4 and 0.5 , respectively.

noted that while calculating error $[\mathrm{Fe} / \mathrm{H}]$, we have not considered the error in metallicity associated with individual calibration points.

The error in metallicity is plotted with respect to the $[\mathrm{Fe} / \mathrm{H}]$ for cut-off criterion IV for both data sets and compared in the Fig. 24. As seen, the error for MCPS (blue) is in the range of $\sim 0.10-0.25$, whereas that for OGLE III (red) is in the range of $\sim 0.15-0.30$. Fig. 25 shows the distribution of error for OGLE III and MCPS data. The distribution appears similar in both cases, except that the 


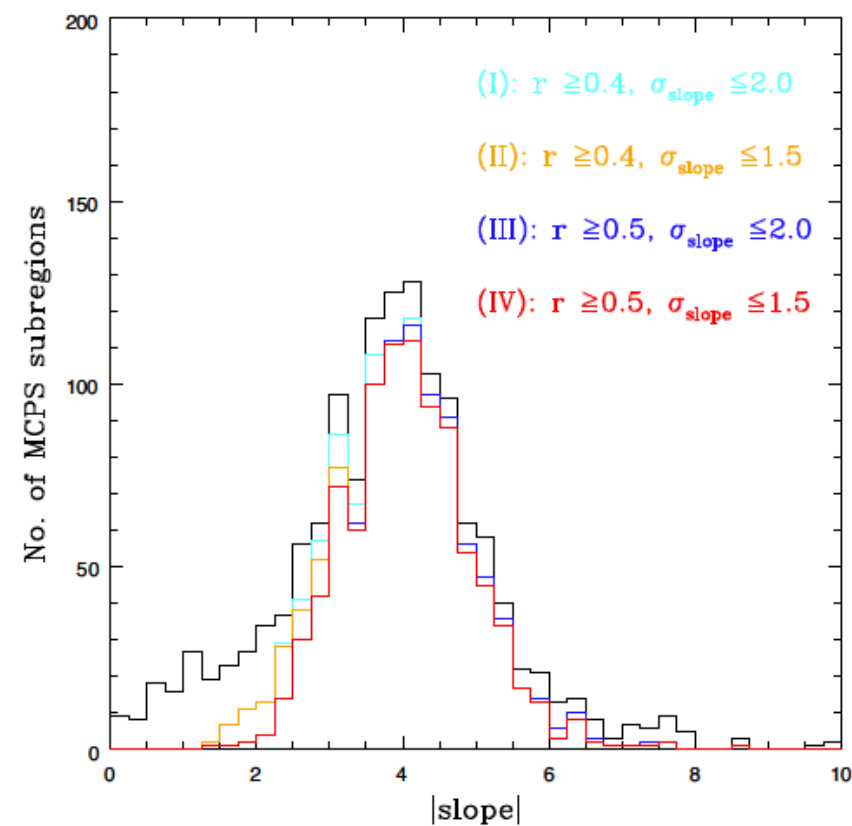

Figure 19. Histogram of |slope| for MCPS sub-regions estimated for all the four cut-off criteria ((I) in cyan, (II) in orange, (III) in blue, and (IV) in red). $N_{p} \geq 10$ for all these four cases. The black solid line shows the distribution of |slope| with no cut-off corresponding to all MCPS sub-regions.

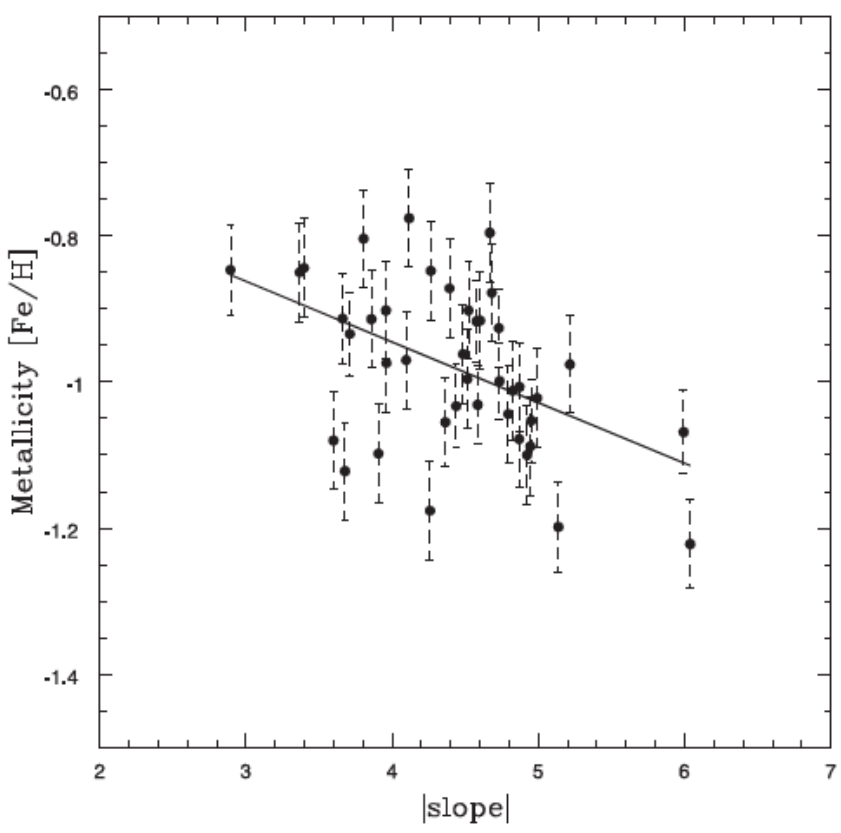

Figure 20. Plot of [Fe/H] versus |slope| for MCPS data. The points denote our sub-regions whose mean [Fe/H] has been found using RGs from Dobbie et al. (2014b), with the solid line denoting a linear relation between them. The error bar (dashed line) shown for each point is the standard error of mean $[\mathrm{Fe} / \mathrm{H}]$.

OGLE III distribution has more regions and is shifted with respect to the MCPS distribution by almost constant value of 0.05 dex.

\section{DISCUSSION}

We have created maps of the photometrically estimated metallicity of the SMC using OGLE III and MCPS data. The two data sets
Table 5. Calibrators for MCPS slope-metallicity relation.

\begin{tabular}{|c|c|c|c|c|c|c|}
\hline $\begin{array}{l}\text { RA } \\
\left({ }^{\circ}\right)\end{array}$ & $\begin{array}{l}\text { Dec. } \\
\left({ }^{\circ}\right)\end{array}$ & |slope| & $\sigma_{\text {slope }}$ & $r$ & $\begin{array}{c}\text { Mean }[\mathrm{Fe} / \mathrm{H}] \\
(\mathrm{dex})\end{array}$ & $\begin{array}{l}\text { Standard error } \\
\text { of mean }[\mathrm{Fe} / \mathrm{H}]\end{array}$ \\
\hline 8.26 & -72.32 & 4.79 & 0.70 & 0.81 & -1.04 & 0.07 \\
\hline 9.25 & -72.15 & 4.92 & 0.51 & 0.84 & -1.10 & 0.07 \\
\hline 9.75 & -71.99 & 4.87 & 0.42 & 0.89 & -1.08 & 0.07 \\
\hline 9.75 & -72.15 & 4.39 & 0.52 & 0.80 & -0.87 & 0.07 \\
\hline 9.76 & -72.32 & 4.73 & 0.43 & 0.86 & -1.00 & 0.05 \\
\hline 10.25 & -71.82 & 5.22 & 0.55 & 0.90 & -0.98 & 0.07 \\
\hline 10.26 & -71.99 & 3.71 & 0.45 & 0.79 & -0.93 & 0.06 \\
\hline 10.26 & -72.15 & 4.87 & 0.59 & 0.82 & -1.01 & 0.06 \\
\hline 10.75 & -71.65 & 4.68 & 0.70 & 0.82 & -0.88 & 0.07 \\
\hline 10.76 & -71.82 & 4.82 & 0.57 & 0.85 & -1.01 & 0.07 \\
\hline 10.75 & -71.99 & 4.99 & 0.50 & 0.88 & -1.02 & 0.07 \\
\hline 18.74 & -72.15 & 5.99 & 0.75 & 0.87 & -1.07 & 0.06 \\
\hline 18.74 & -72.32 & 4.58 & 0.62 & 0.79 & -1.03 & 0.05 \\
\hline 8.76 & -72.48 & 4.58 & 0.48 & 0.86 & -0.92 & 0.06 \\
\hline 7.75 & -74.14 & 4.09 & 0.46 & 0.84 & -0.97 & 0.07 \\
\hline 8.75 & -74.14 & 3.60 & 0.48 & 0.77 & -1.08 & 0.07 \\
\hline 15.25 & -74.15 & 4.26 & 0.39 & 0.84 & -1.18 & 0.07 \\
\hline 19.75 & -73.65 & 3.91 & 0.87 & 0.73 & -1.10 & 0.07 \\
\hline 10.75 & -74.65 & 3.68 & 0.65 & 0.74 & -1.12 & 0.07 \\
\hline 13.25 & -74.48 & 4.44 & 0.35 & 0.88 & -1.03 & 0.06 \\
\hline 13.75 & -74.48 & 5.14 & 0.54 & 0.82 & -1.20 & 0.06 \\
\hline 13.74 & -74.65 & 4.95 & 0.53 & 0.86 & -1.05 & 0.06 \\
\hline 15.24 & -74.81 & 6.04 & 0.82 & 0.90 & -1.22 & 0.06 \\
\hline 11.88 & -72.15 & 4.26 & 0.44 & 0.83 & -0.85 & 0.07 \\
\hline 12.88 & -71.99 & 3.96 & 0.55 & 0.72 & -0.90 & 0.07 \\
\hline 13.13 & -71.82 & 4.36 & 0.38 & 0.93 & -1.05 & 0.06 \\
\hline 14.88 & -71.65 & 4.73 & 0.63 & 0.84 & -0.93 & 0.05 \\
\hline 16.87 & -71.99 & 4.12 & 0.80 & 0.76 & -0.78 & 0.07 \\
\hline 8.37 & -72.99 & 3.86 & 0.44 & 0.86 & -0.91 & 0.07 \\
\hline 10.38 & -72.49 & 4.51 & 0.41 & 0.87 & -1.00 & 0.07 \\
\hline 18.12 & -72.65 & 4.48 & 0.52 & 0.88 & -0.96 & 0.07 \\
\hline 10.87 & -73.82 & 3.80 & 0.39 & 0.84 & -0.80 & 0.07 \\
\hline 13.25 & -71.99 & 4.67 & 0.66 & 0.80 & -0.80 & 0.07 \\
\hline 16.75 & -72.48 & 3.96 & 0.63 & 0.76 & -0.97 & 0.07 \\
\hline 16.75 & -72.65 & 3.66 & 0.62 & 0.78 & -0.91 & 0.06 \\
\hline 12.25 & -73.65 & 3.40 & 0.44 & 0.74 & -0.84 & 0.07 \\
\hline 10.37 & -72.86 & 3.37 & 0.51 & 0.72 & -0.85 & 0.07 \\
\hline 10.13 & -73.11 & 4.94 & 0.63 & 0.84 & -1.09 & 0.07 \\
\hline 16.62 & -72.77 & 4.52 & 0.89 & 0.76 & -0.90 & 0.07 \\
\hline 11.87 & -73.44 & 2.89 & 0.42 & 0.73 & -0.85 & 0.06 \\
\hline 12.63 & -73.52 & 4.60 & 0.87 & 0.71 & -0.92 & 0.07 \\
\hline
\end{tabular}

Note: The table lists out the 41 calibrators used to construct the slopemetallicity relation for MCPS data. The column descriptions are same as Table 2.

are complementary in terms of the area coverage of the SMC. The method traces the densest part of the RGB and the slope is estimated using a straight line fit. We thus estimate only the metallicity of the population that has the largest number of stars in the RGB phase. The analysis does not bring out any information regarding the contribution from the less dominant populations. We estimated the slope of the RGB of several sub-regions within the SMC using this method that takes care of reddening and density variation between regions. The RGB slope is then converted to metallicity using spectroscopic measures that nearly covers the full range. If there are multiple populations with similar number of stars, then the RGB tends to be broad and the slope may be poorly estimated. These regions are not considered in our analysis, due the removal of regions with poor estimation of slope. We discuss the assumptions made in this study and the implications of our results in the following sections. 


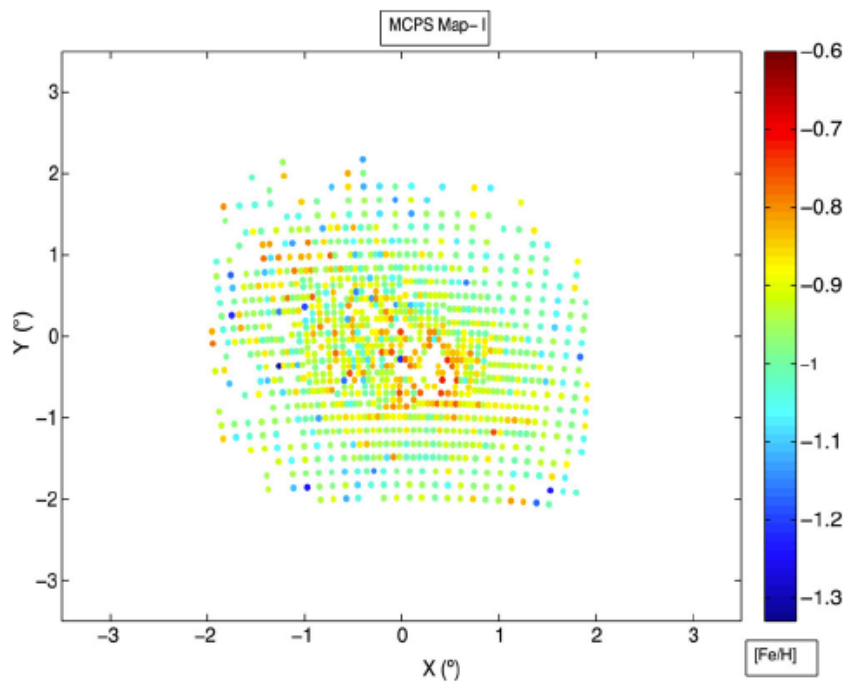

Figure 21. MCPS metallicity map with cut-off criterion (I): $N_{p} \geq 10, r \geq$ 0.4 , and $\sigma_{\text {slope }} \leq 2.0$.

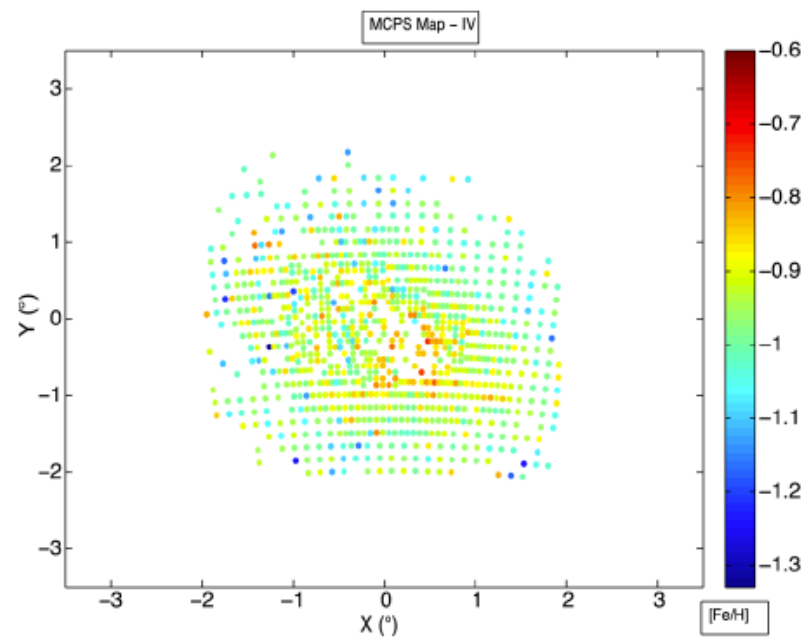

Figure 22. MCPS metallicity map with cut-off criterion (IV): $N_{p} \geq 10$, $r \geq 0.5$, and $\sigma_{\text {slope }} \leq 1.5$.

\subsection{Assumptions and their impact}

The steps undertaken to create the metallicity maps might have affected the outcome of the study. Below we discuss each step and its impact on the estimated value of RGB slope and metallicity.

\subsubsection{Effect of sub-division}

The two data sets are spatially sub-divided to create regions with smaller area. We have also considered different sizes for subdivision based on the stellar density. As the depth and resolution of MCPS and OGLE III are different, we were unable to make the sub-regions the same area in both data sets. The MCPS sub-regions are relatively larger than the OGLE III sub-regions throughout the SMC. As a result, even though we bring them to the same metallicity scale, we are unable to make one-to-one matches between the metallicity estimated using the two data sets. As the area of a subregion changes, the estimated slope changes mildly, though within the errors. This may be due to the fact that the dominant population as well as differential reddening can change with area.

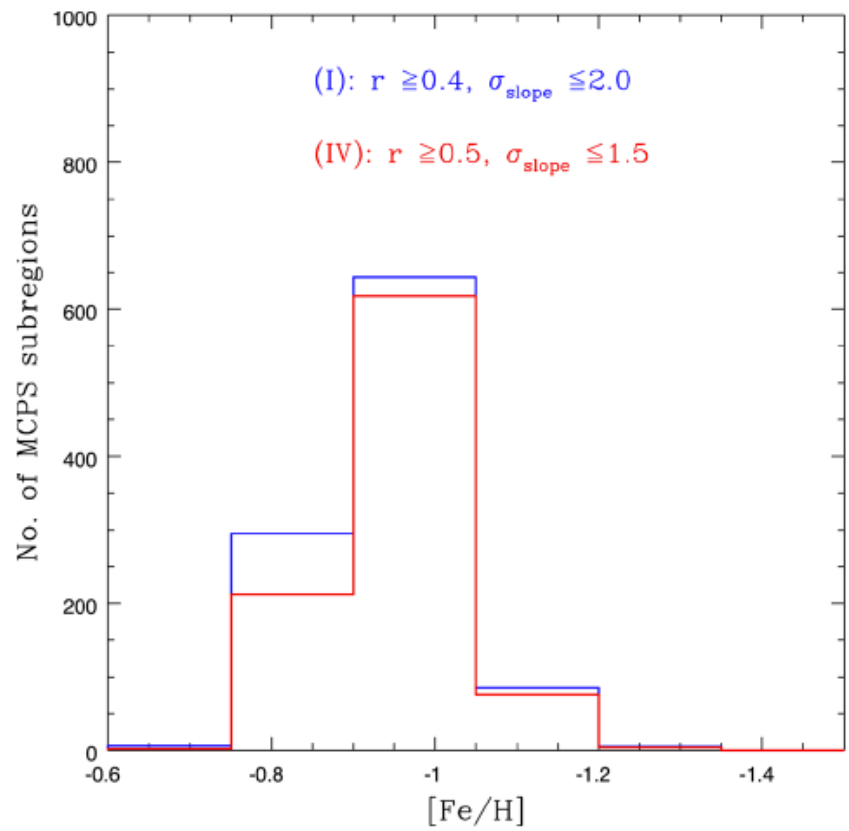

Figure 23. Histogram of $[\mathrm{Fe} / \mathrm{H}]$ for MCPS data, estimated for cut-off criteria (I) in blue and (IV) in red. $N_{p} \geq 10$ for both cases.

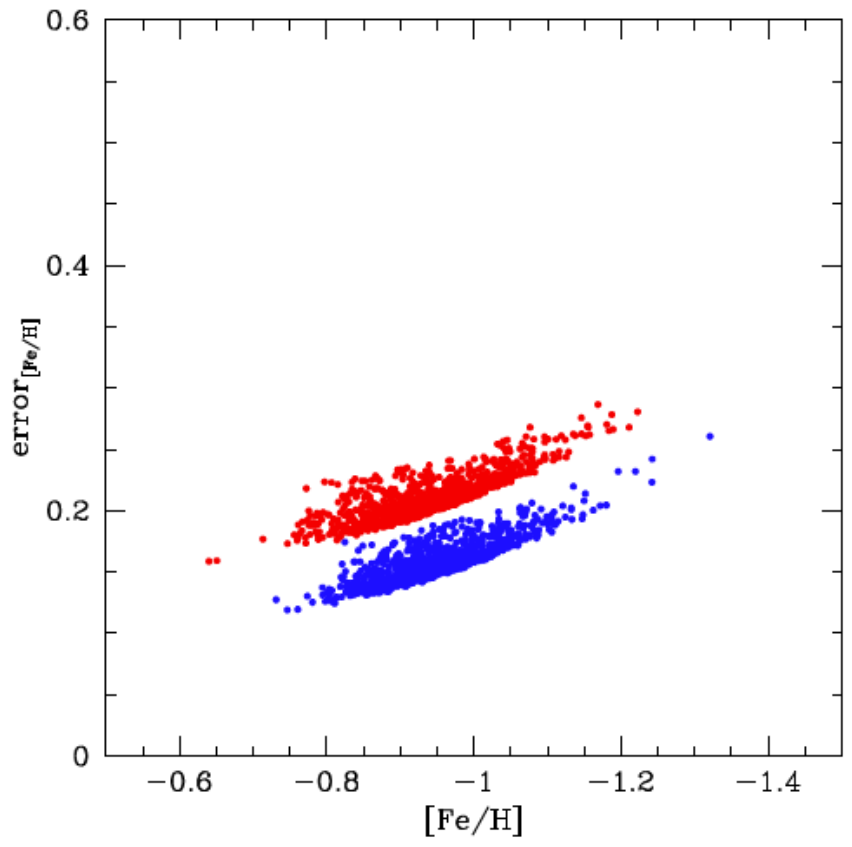

Figure 24. Plot of error ${ }_{[\mathrm{Fe} / \mathrm{H}]}$ versus [Fe/H] for OGLE III (red filled circles) and MCPS (blue filled circles) for cut-off criterion (IV).

\subsubsection{Effect of reddening and differential reddening}

In the reddening map presented by Subramanian \& Subramaniam (2012) using RC stars, the SMC is shown to have variation in reddening across the galaxy. Also, the south-west and north-east regions about the SMC centre and the Eastern Wing region have larger reddening compared to other regions within the SMC. The reddening variation can shift the location of the RGB in the CMD. This effect is taken care in the analysis by anchoring the RGB to the densest part of the RC. But, it is to be noted that the effect of differential reddening will remain and make the RGB broad. 
Table 6. Mean metallicity for SMC using MCPS data.

\begin{tabular}{lccccc}
\hline Cut-off criteria & $r$ & $\sigma_{\text {slope }}$ & Region of the SMC & Number of sub-regions & Mean [Fe/H] (dex) \\
\hline I & $\geq 0.40$ & $\leq 2.0$ & COMPLETE & 1035 & $-0.94 \pm 0.08$ \\
IV & $\geq 0.50$ & $\leq 1.5$ & COMPLETE & 913 & $-0.95 \pm 0.07$ \\
\hline
\end{tabular}

Note. The column descriptions are same as Table 3, except that the fourth column denotes complete coverage for MCPS data.

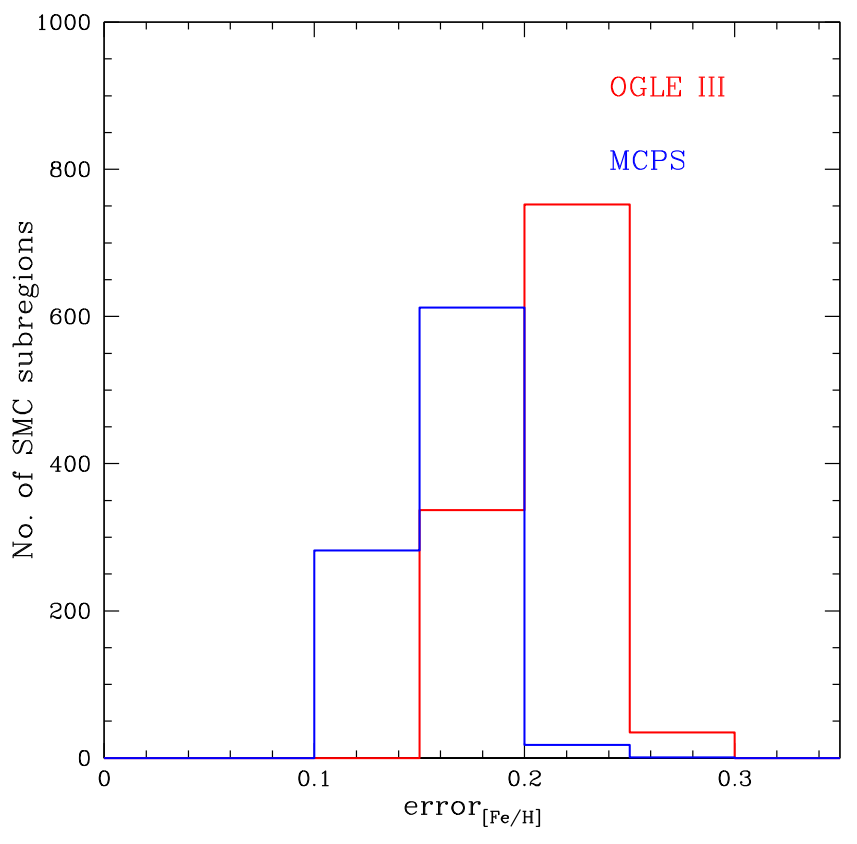

Figure 25. Plot of histogram of error $[\mathrm{Fe} / \mathrm{H}]$ for OGLE III (red) and MCPS (blue) for cut-off criterion IV.

Large-scale variation of reddening can broaden the RGB, resulting in a poorly estimated slope. Such regions are eliminated from our analysis. Most of the regions that get eliminated due to poor slope estimation are found to be located near the regions with large variation in reddening (around the centre, and Eastern Wing). Since, we are interested in the statistical average estimated using large number of regions, their impact is likely to be negligible.

\subsubsection{Effect of line of sight depth}

The SMC is known to have large LOS depth as traced by young, intermediate-age, and old stellar populations (Mathewson, Ford \& Visvanathan 1986, 1988; Crowl et al. 2001; Subramanian \& Subramaniam 2012). Recently, Subramanian et al. (2017) identified regions towards east of $\sim-2^{\circ}$ that show a foreground population $(\sim 11.8 \pm 2.0 \mathrm{kpc}$ in front of the main body) in the form of a distance bimodality in the RC distribution. The authors analysed a larger area $\left(\sim 20 \mathrm{deg}^{2}\right)$ than our observed region using near-infrared photometric data from the VISTA-VMC survey. They relate the bimodality in their extreme east regions to tidal stripping from the SMC during the most recent encounter with the LMC. The OGLE III observed field has a very few sub-regions $(<4$ per cent of analysed sub-regions) beyond $\sim-2^{\circ}$ east, whereas the MCPS has none. The tip of the RGB might vary in sharpness, being more spread out where the LOS depth is large, which may affect the estimation of slope by making the RGB appear steeper than it otherwise would for a given metallicity. Also, the RGB in these extreme east regions are sparsely populated as they lie at the periphery of the observed region. These factors can lead to poor estimation of RGB slope. Thus, the effect on our results by such sub-regions will be negligible. This is because, they either get excluded by cut-off criteria, or their percentage is insignificant compared to the total number of sub-regions analysed. Subramanian et al. (2017) also mention the presence of a very mild gradient in distance modulus from east to west of the SMC (between $-2^{\circ}$ and $2^{\circ}$ ), and no gradient as such from north to south. However, according to Subramanian \& Subramaniam (2009, 2012) who used the RC distribution from MCPS and OGLE (II and III) data to estimate the LOS depth of the SMC, it was found to that the LOS depth is almost uniform across the inner SMC. Thus, we are consistent with our method in estimating the slope of RGB and calibrating the same to metallicity within our observed area. In this study, we are interested in the mean feature of the RGB. So, for mild/uniform variation in depth within a region the effect of RGB spread on our result will be mild/unlikely. The authors point out that the regions around the SMC centre and north-east suffer from a larger LOS depth. These regions with large depth get removed from our map due to poor slope estimation using the cut-off criteria on the estimated parameters.

\subsubsection{Systematics in the calibration}

There can be a systematic effect in our study related to the conversion of slope to metallicity. We have tried to minimize it by formulating independent slope-metallicity relation for OGLE III and MCPS data using the same spectroscopic study. Across the inner $2.5 \mathrm{deg}$ of the SMC, the RGB slope has a range of values; the calibration relation between slope and metallicity should hold good for the full range. We used the result of Dobbie et al. (2014b) that has a large range in metallicity to achieve this calibration independently for both data sets. The calibration of RGB slope to metallicity rests on the assumption that the spectroscopic targets are drawn from the dominant population of the sub-region. This assumption is fair enough, as the spectroscopic targets are also chosen from the RGB and are likely to be picked from the population with most number of stars.

\subsection{Comparison of metallicity distribution}

\subsubsection{Mean metallicity of the SMC}

In Fig. 26, we compare the distribution of metallicity for the entire SMC as estimated from these two data sets. It can be seen that in general the distributions are almost identical. The MCPS distribution has primary and secondary peaks at same metallicity values as OGLE III. The difference in the peak heights are due to the difference in number of sub-regions analysed for the two surveys. If we approximate the distribution to be Gaussian, then, for the OGLE III data, the peak of the distribution is estimated to be at $[\mathrm{Fe} / \mathrm{H}]=-0.94(\sigma[\mathrm{Fe} / \mathrm{H}]=0.09)$. In the case of MCPS data, the 


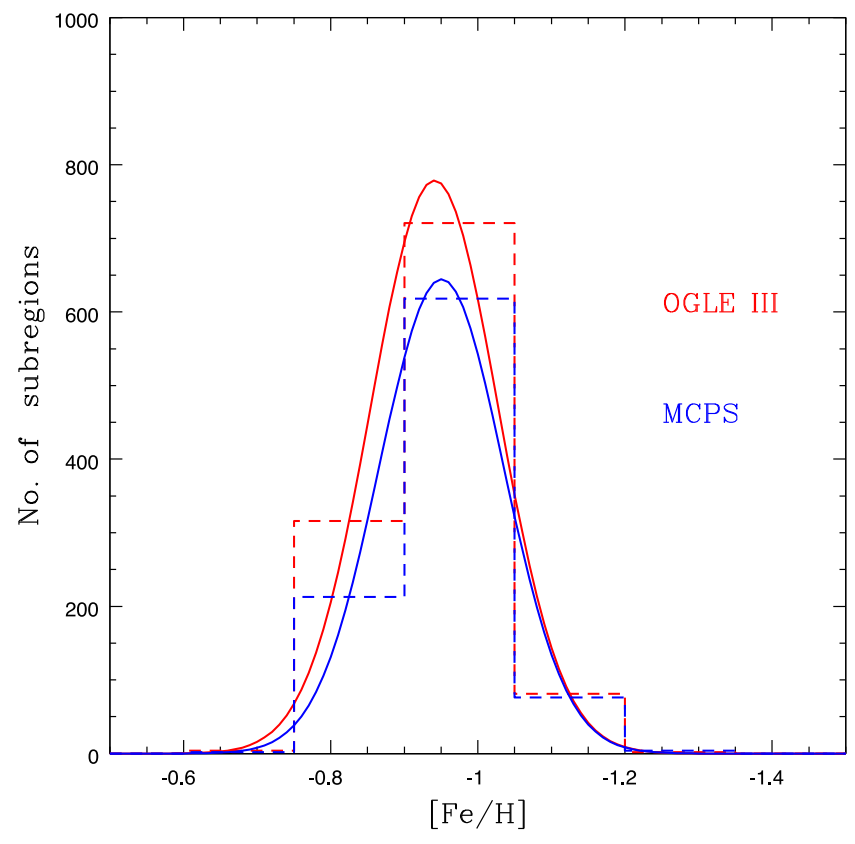

Figure 26. Histogram of metallicity (dashed lines) for the complete SMC fitted with a Gaussian function (solid lines) for cut-off criterion IV of OGLE III (red) and MCPS data (blue).

peak of the distribution is found to be $[\mathrm{Fe} / \mathrm{H}]=-0.95(\sigma[\mathrm{Fe} / \mathrm{H}]=$ 0.08 ). Similar peak values and widths for both data sets suggest that the difference in area coverage does not have significant role in the derived metallicity distribution of the SMC. In other words, the northern and southern SMC seen in the MCPS do not have much variation in metallicity, which varies between $\approx-0.9$ and 1.0 dex. The range of metallicity within the SMC is found to be relatively less $(\approx-0.75$ dex to -1.2$)$ as compared to that found in the LMC in Paper I $(\approx-0.2$ dex to -0.75 dex, fig. 36$)$. An important point to be noted here is that, for the LMC in the MCPS analysis, the I magnitude of MCPS data was first transformed to that of OGLE III data, and then the re-estimated MCPS slopes were transformed to metallicity using the OGLE III slope-metallicity relation. However, in this work, we have been able to estimate independent slope-metallicity relations for both OGLE III and MCPS data sets, respectively. Thus, there does not exist any effect in our metallicity estimations due to systematic differences between the OGLE III and MCPS filter systems.

Our results for the mean metallicity of the SMC is in agreement with that of Carrera et al. (2008), Parisi et al. (2010, 2016), and Dobbie et al. (2014b). Carrera et al. (2008) performed CaT spectroscopy to obtain metallicities of a sample of some 350 field red giants in 13 fields of size $(8.85 \times 8.85) \operatorname{arcmin}^{2}$ distributed from $\sim 1^{\circ}$ to $4^{\circ}$ from the centre. They found a mean metallicity of $[\mathrm{Fe} / \mathrm{H}] \sim-1.0 \mathrm{dex}$ in the innermost SMC fields $\left(<2.5^{\circ}\right)$. Parisi et al.'s group have a series of work using CaT spectroscopy of RGs within the SMC field region (Parisi et al. 2010, 2016) and star clusters (Parisi et al. 2009, 2015). Parisi et al. (2010) estimated the metallicities of $\sim 360$ red giant stars distributed in $15 \mathrm{SMC}$ fields from the centre till about $8^{\circ}$. The metallicity distribution of their whole sample had a mean value of $[\mathrm{Fe} / \mathrm{H}]=$ $-1.00 \pm 0.02 \mathrm{dex}$, with a dispersion of $0.32 \pm 0.01$. However, the derived mean values for fields within $4^{\circ}$ radius was about $[\mathrm{Fe} / \mathrm{H}]=-0.99 \pm 0.08 \mathrm{dex}$, whereas that beyond $4^{\circ}$ was $[\mathrm{Fe} / \mathrm{H}]=$
$-1.02 \pm 0.07$ dex. Later, Parisi et al. (2016) analysed a sample of 400 RGs within 15 fields, thus increasing their total sample of field stars to $\sim 750$. The authors reported a median metallicity for this sample to be $-0.97 \pm 0.01$ dex. The authors Parisi et al. (2015) using a combined sample of clusters from previous works yielded a high probability that the metallicity distribution for clusters is not uni-modal as the field stars but bi-modal, with peaks at -1.1 and -0.8 dex. Although we have used Dobbie et al. (2014b) to calibrate our metallicity map, we would like to state that our estimated mean metallicities using two large-scale data sets are in accordance with their study. According to them, the metallicity distribution of their field star sample distributed within $5^{\circ}$ of SMC centre has a median metallicity of $-0.99 \pm 0.01 \mathrm{dex}$, which is similar to the mean obtained in this study.

The mean metallicity of the SMC estimated by us is metalrich as compared to previous studies using RR Lyraes by Deb \& Singh (2010), Haschke et al. (2012), and Kapakos \& Hatzidimitriou (2012), where the estimated mean is $\leq-1.50 \mathrm{dex}$. Given that we use RGB stars as indicators, the difference between our results is possibly related to the mean age difference between the RGBs and RR Lyraes. Due to mass and metallicity effects on RGB evolutionary rates, it is difficult for old, metal-poor populations to be the dominant contributor to the bulk average metallicity of red giants (Manning \& Cole 2017).

\subsubsection{Metallicity gradient of the SMC}

Next, we try to understand the metallicity distribution as a function of radius. To do so, the orientation of the SMC and projection effects must be addressed. The structure of the SMC is complex and less understood as compared to the LMC. According to theoretical and observational studies (Zaritsky et al. 2000; Harris \& Zaritsky 2006; Bekki \& Chiba 2008; Evans \& Howarth 2008), the SMC is supposed to be a two-component system, where old- and intermediate-age stars are distributed in a spheroidal or slightly ellipsoidal component, whereas the young stars and gas are distributed in a disc.

Subramanian \& Subramaniam (2012) studied the old RR Lyrae stars and the intermediate-age RC stars in the SMC and suggested that both these populations have a slightly ellipsoidal distribution and are located in a similar volume. The smooth ellipsoidal distribution of RR Lyraes is also demonstrated by recent works of Jacyszyn-Dobrzeniecka et al. (2017) and Muraveva et al. (2018) from analysis of large-area survey of the SMC in optical (OGLE IV) and near-infrared (VISTA-VMC) bands, respectively. However, contrary to previous belief on the distribution of younger populations, Jacyszyn-Dobrzeniecka et al. (2016) from their analysis Classical Cepheids using OGLE-IV data demonstrated the existence of a non-planar structure that can be described as an extended ellipsoid. Scowcroft et al. (2016) using the mid-IR data of Cepheids from Spitzer examined the three-dimensional structure of the SMC. The authors confirm that the Cepheid distribution does not just have a large line-of-sight depth, but is elongated from the northeast to the south-west, such that the south-western side is up to $20 \mathrm{kpc}$ more distant than the north-east. Also, very recently Ripepi et al. (2017) using near-infrared data (VISTA-VMC) show that the three-dimensional distribution of the Classical Cepheids is not planar, but heavily elongated for more than $25-30 \mathrm{kpc}$ in the east/northeast towards south-west direction (approximately). Thus, the structure of the SMC remains a debated topic. It is also believed that the SMC is markedly elongated along the line of sight (Gardiner \& Hawkins 1991; Haschke et al. 2012; Subramanian \& Subramaniam 

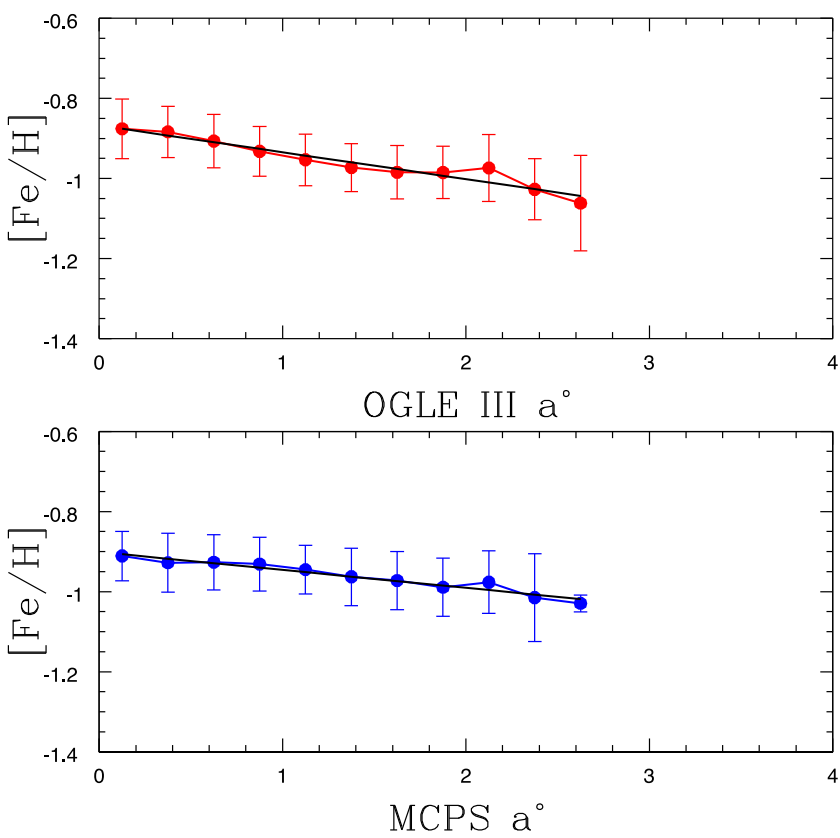

Figure 27. Variation of metallicity with semimajor axis (a) of the SMC for OGLE III (top panel) and MCPS (bottom panel) data, estimated for cut-off criterion IV. The MG estimated till $\sim 2.5^{\circ}$ is shown as black solid line for each panel.

2012; Jacyszyn-Dobrzeniecka et al. 2016), making projection effects important but the determination of true galactocentric distances difficult to ascertain.

We have estimated the radial MG following the convention used by previous studies by Piatti et al. (2007a), Parisi et al. (2009), Parisi et al. (2010), and Dobbie et al. (2014b). This is shown in Fig. 27. For this, we considered an elliptical system whose major axis is positioned along the SMC bar (i.e. along north-east-southwest). We adopted this major axis to have an position angle of $55.3^{\circ}$ east of north. If ' $a$ ' is the semimajor and ' $b$ ' is the semiminor axis of the ellipse, we assumed the ratio $a / b=1.5$ [as mentioned in Dobbie et al. (2014b) in accordance with Subramanian \& Subramaniam (2012)]. For each star, we then estimated the value of ' $a$ ', that an ellipse would have if it were centred on the SMC, aligned with the bar, and one point of its trajectory coincided with star's position. This value of ' $a$ ' is used as a surrogate for the true galactocentric distance. We constructed a radial MG, by assuming a bin width of $0.25^{\circ}$, and fitting a straight line using least-squares fit. The radial MG estimated for OGLE III is estimated to be $[\mathrm{Fe} / \mathrm{H}]=$ $(-0.868 \pm 0.009)+a \times(-0.067 \pm 0.006), r=0.97$; for MCPS, it is $[\mathrm{Fe} / \mathrm{H}]=(-0.901 \pm 0.006)+a \times(-0.045 \pm 0.004), r=0.97$. The unit of the MG is dex deg ${ }^{-1}$.

The estimated MG in the SMC is shallow and in accordance with that of previous studies by Carrera et al. (2008), Parisi et al. (2016), and Dobbie et al. (2014b). We present a qualitative comparison between our results and some previous studies. Although Carrera et al. (2008) had mentioned the MG as one moves away from centre to larger galactocentric distances, their mean metallicities remain almost constant $(\sim-1$ dex $)$ till $2.5^{\circ}$ from the centre and drops to metal-poor values $(\sim-1.6 \mathrm{dex})$ only for two outer fields located between $2.5^{\circ}-4^{\circ}$. The values for these two fields are also associated with relatively larger error. It needs a mention that Carrera et al.'s results are based on fields distributed in the south, east, and western regions, and none in the northern part of the SMC. The authors do not quantify the MG and do not consider an elliptical geometry.

Parisi et al. (2010) in their initial work claimed the non-existence of an MG. With their latest refined work, Parisi et al. (2016) observed an MG in a larger sample of RGs. Their fig. 1 shows that their sample covers small pockets within the SMC except the north-west portion. While estimating the MG, they considered elliptical geometry (but with $b / a=1 / 2)$ and within the inner region of the galaxy $\left(a<4^{\circ}\right)$ found a clear MG for the field stars of $-0.08 \pm 0.02 \mathrm{dex} \mathrm{deg}^{-1}$. On the other hand, in the outer part of the SMC $\left(a>4^{\circ}\right)$ the authors report a positive MG, although the authors mark a word of caution that it requires detailed study to confirm this positive MG. We could not confirm this positive MG due the limitation in our spatial coverage.

The MG based on clusters has a different story. The combined cluster samples from Parisi et al. (2009, 2015), Da Costa \& Hatzidimitriou (1998), and Glatt et al. (2008a,b) show that for a distance less than $4^{\circ}$, they appear to be concentrated in two groups: one metal-rich group and the other metal-poor group relative to the field stars. The gradients of these two groups (metal-poor group: $-0.03 \pm 0.05 \mathrm{dex} \mathrm{deg}{ }^{-1}$, with $Y$-intercept at $\approx-1.1 \mathrm{dex} ;$ metal-rich group: $-0.01 \pm 0.02 \mathrm{dex} \mathrm{deg}^{-1}$, with $Y$-intercept at $\approx-0.75 \mathrm{dex}$ ) are shallow and extremely dependent on the assumed vertex and on the edge definition made between the inner and outer regions. Also, the statistical significances of these two potential gradients are difficult to assess.

Dobbie et al. (2014b) considered similar geometry as this work and estimated the MG to be $-0.075 \pm 0.011 \mathrm{dex} \mathrm{deg}^{-1}$ within the inner $5^{\circ}$. The authors had covered a considerable space of about $37.5 \mathrm{deg}^{2}$ about the SMC centre in all directions. However, since the authors dealt with individual stars, a lot of regions within the inner SMC were missed out for which we could estimate the mean metallicity. Overall, we see that the gradients estimated by Parisi et al. (2016) and Dobbie et al. (2014b) seem a little steeper as compared to that estimated by us but are similar within the errors. The reasons could be the following: (1) we are estimating the mean metallicity of individual sub-regions and not stars, and then further smudging the mean metallicity while estimating the radial MG. (2) Our study is limited to $\sim 2.5^{\circ}$, whereas their work extends till $4^{\circ}-5^{\circ}$. There can be an actual dip in mean metallicity beyond $2.5^{\circ}$ that can cause the MG to go down. In that case, one needs to extend our technique using to the outer layers of the galaxy in order to understand the true nature of MG. (3) Our data sets have a more homogeneous distribution in space as compared to the above studies. Kapakos \& Hatzidimitriou (2012) estimated a very shallow, tentative MG $\left(-0.013 \pm 0.007 \mathrm{dex} \mathrm{kpc}^{-1}\right)$ in the deprojected plane of the SMC, by analysing $V$-band light curves of 454 RR Lyraes using OGLE III data. However, as mentioned by the authors, the MG could be affected by selection effects and the confirmation of MG will require a detailed spectroscopic investigation.

Our results of MG are not similar to that of Cioni (2009), who using the AGBs estimated that the $[\mathrm{Fe} / \mathrm{H}]$ has a constant value of $\sim-1.25 \pm 0.01$ dex from centre up to $\sim 12 \mathrm{kpc}$. The origin of this discrepancy may be due to the difference in mean age between the general RGB field discussed here and AGB stars previously studied. The existence of MG is also in disagreement with Piatti (2012) who mentioned that there does not exists any metallicity or age gradient within the SMC, and with Haschke et al. (2012) and Deb et al. (2015) who do not detect any MG from the analysis RR Lyrae stars.

According to the studies of Da Costa \& Hatzidimitriou (1998), Idiart, Maciel \& Costa (2007), Carrera et al. (2008), and Cignoni et al. (2013), the most metal-rich stars within the SMC tend to be 

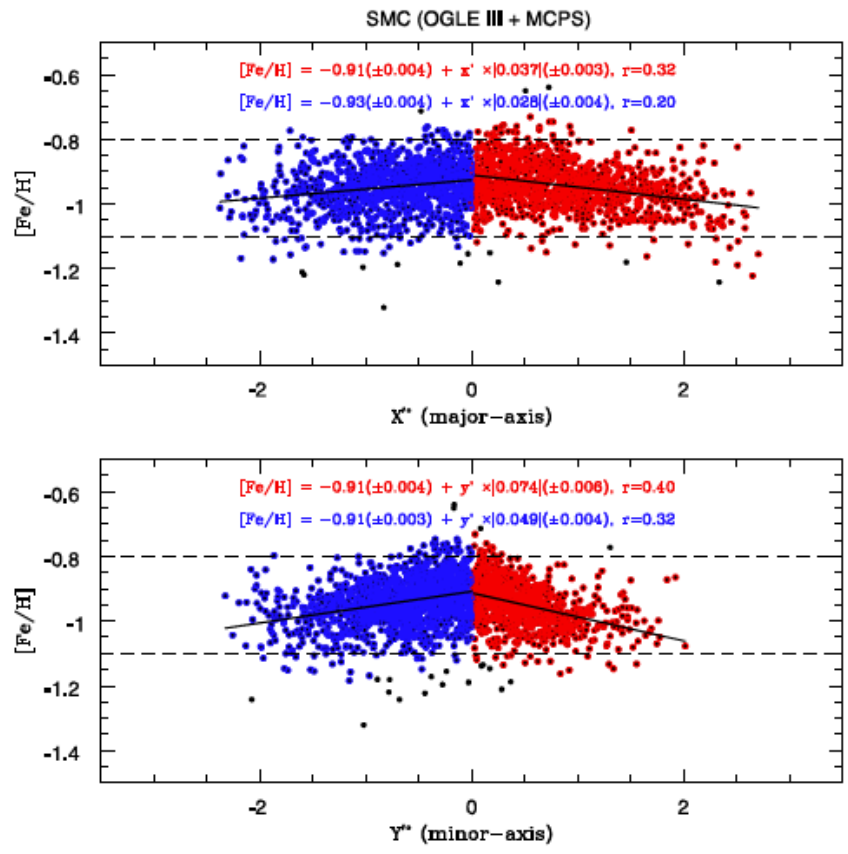

Figure 28. Estimated MG along the major (top panel) and minor (bottom panel) axis. The positive major axis is along south-west direction and the negative is along north-east, whereas the positive minor axis is along northwest direction and the negative is along south-east direction. The combined data points (OGLE III and MCPS - cut-off criterion IV) are plotted as black open circles in both panels. The red open circles denote points after estimating MG with $3 \sigma$ clipping along the positive major and minor axis, whereas the blue open circles denote points after estimating MG with $3 \sigma$ clipping along the negative major and minor axis. The estimated MG, $Y$ intercept, and $r$ are mentioned in both panels corresponding to each case.

the youngest. Carrera et al. (2008) found a relationship between the MG and the age gradient, in the sense that the youngest stars are concentrated in the central metal-rich regions. Piatti (2011) from his study of SMC star clusters established an age-metallicity relation, where they found a decrease from $[\mathrm{Fe} / \mathrm{H}] \sim-0.5$ to $-1.0 \mathrm{dex}$ for the 1-2-Gyr-old populations down to typically $[\mathrm{Fe} / \mathrm{H}]=-1.0$ to -1.5 dex for the populations older than 5-6 Gyr. The author inferred that the younger population within the SMC is more concentrated towards the centre of the galaxy.

Cignoni et al. (2013) and Rubele et al. (2015) while analysing the star-formation history of the SMC field showed that the bar region and Wing region of the SMC have undergone recent star formation and host younger population. Moreover, Cignoni et al. (2013) mentioned that for similar age they do not observe any MG. Dobbie et al. (2014b) and Parisi et al. (2016) assign their detection of MG to this increasing fraction of young stars in the inner regions of the SMC. From our analysis of a homogeneous data set covering the inner SMC, we infer that the shallow but gradual MG that we detect in the inner SMC is possibly due to similar effect.

We plotted the combined metallicity distribution of OGLE III and MCPS data sets with respect to the major and minor axis of the SMC to check for any possible gradients along them. Fig. 28 shows the variation of metallicity (black points) along the major and minor axes in the top and bottom panels, respectively. The positive major axis is along south-west direction and the negative is along north-east direction, whereas the positive minor axis is along north-west direction and the negative is along south-east direction.
The metallicity range, in general, is seen to be constrained between -0.80 and -1.10 dex for both data sets (with some deviations), which is $\pm 1 \sigma$ about the mean metallicity $(\approx-0.95 \mathrm{dex})$. These limits are shown by the dashed (black) lines parallel to the major/minor axis. There seems to exist a marginal MG along the major axis, which is shallower relative to the gradient observed along the minor axis.

To quantify the gradients, we made a linear least-squares fit with $3 \sigma$ clipping along the positive (red points) and negative (blue points) major and minor axes. The estimates are labelled within the figures, where the MGs are denoted by their absolute values 0.037 and $0.028 \mathrm{dex} \mathrm{deg}^{-1}$ along positive and negative major axis, respectively, and 0.074 and $0.049 \mathrm{dex} \mathrm{deg}^{-1}$ along positive and negative minor axis, respectively. The MGs along the minor axis are very similar to our estimated radial MG. The correlation coefficient of the fits are also expressed by the absolute value of $(r)$. The MGs estimated in this section indicate that it is not radially symmetric. The spread in metallicity values is more along the negative major and minor axes as compared to their positive counterpart. This is also reflected in $r$ being less along the negative major and minor axes as compared to their positive counterpart. In another words, in the north-east and south-east directions, the metallicity is more dispersed spatially as compared to the north-west and south-west directions. This is possibly because the eastern side of the SMC is more perturbed due to its interaction with the LMC.

\subsection{Spatial distribution of metal-rich regions within the SMC}

In Fig. 29, we present a spatial plot from the combined metallicity map of the LMC and the SMC using both OGLE III and MCPS data sets (for cut-off criterion IV). The former is from fig. 40 of Paper I, where the outliers, i.e. significantly metal-rich and metal-poor region with respect to the mean metallicity of the $\mathrm{LMC}([\mathrm{Fe} / \mathrm{H}] \approx$ $-0.4 \mathrm{dex}$ ), are highlighted in red and green, respectively. The significantly metal-rich regions are distributed primarily along the LMC bar and have $[\mathrm{Fe} / \mathrm{H}] \geq-0.15 \mathrm{dex}$, whereas the metal-poor counterparts have $[\mathrm{Fe} / \mathrm{H}]$ in the range of -0.65 dex to -0.95 dex. Olsen et al. (2011) in their work had reported a population of tidally accreted SMC stars in the outer regions of the LMC. However, we would like to remind the readers that there is no spatial correlation between the accreted population and the metal-poor outliers as reasoned out in Paper I. To indicate the position of the MB, we have shown its main portion as observed by the OGLE IV survey as analysed by Skowron et al. (2014). The centres of the LMC and the SMC are marked by magenta-coloured diamonds. The centre of the SMC eastern Wing is shown as magenta asterisk (Rubele et al. 2015).

We find that the mean metallicity ranges near the central regions of the SMC ( $\approx-0.6$ to -0.85 dex) is very similar to the metal-poor outlier in the LMC. We have highlighted these metal-rich regions within the SMC in blue colour. We see that such regions are mostly spread around the centre along the SMC's major axis (i.e. along the bar). There also exists a scattered distribution of such regions in the SMC outskirts. These are more in the north-east region (in the direction of MB and Wing) when compared to the south-west direction. However, to connect this relatively large spread along the eastern direction to the evolution and interaction history of the SMC requires a detailed spectroscopic and kinematic analysis. The metal-rich regions in the south-west part spatially overlap with the tentatively identified, metal-rich, kinematic counter-bridge structure in Dobbie et al. (2014a,b). 


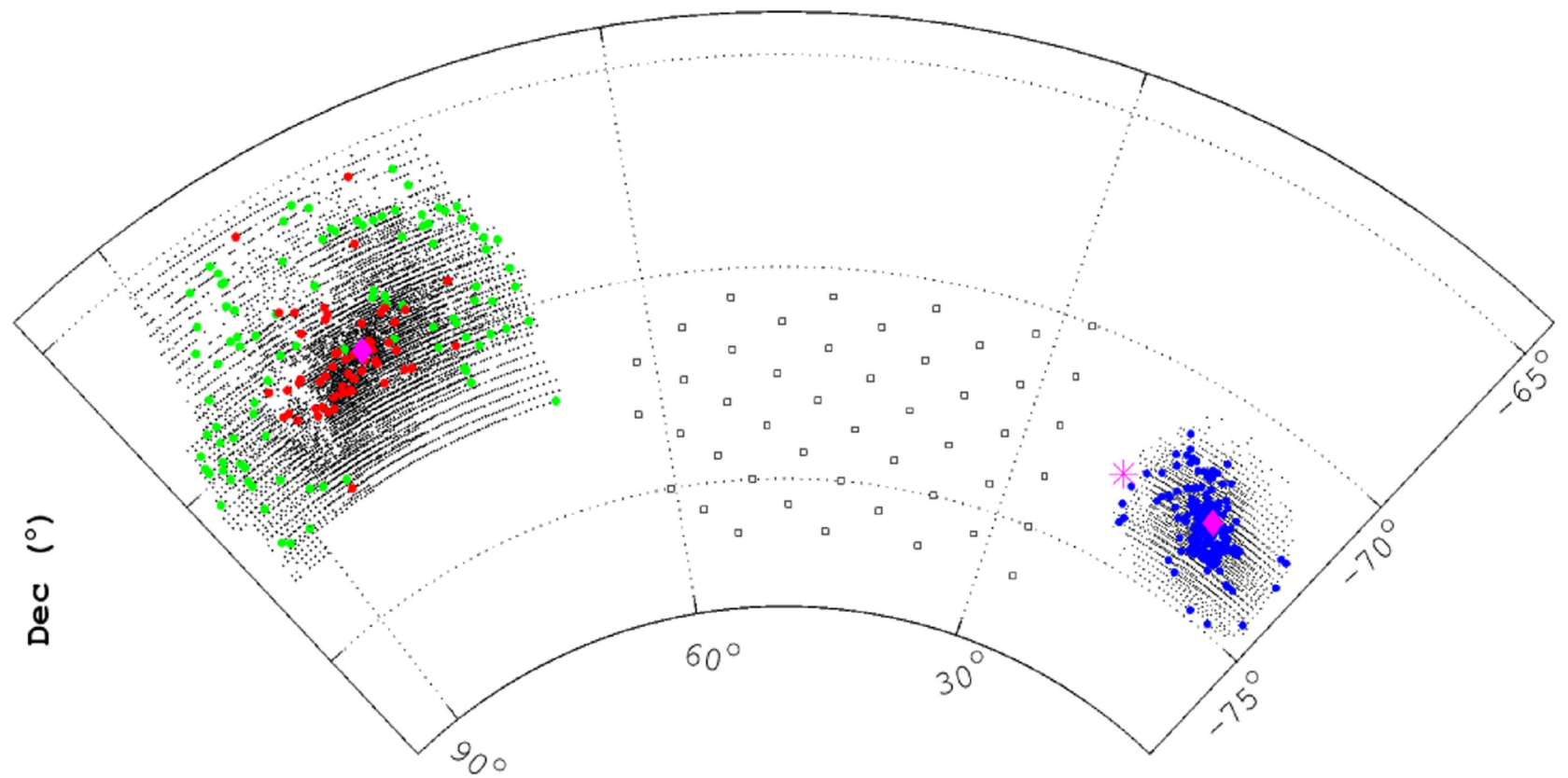

RA $\left({ }^{\circ}\right)$

Figure 29. Combined metallicity map of the MCs using OGLE III and MCPS data for cut-off criterion IV. For the LMC, the metallicity outlier map from fig. 40 of Paper I is shown. The significantly metal-rich $([\mathrm{Fe} / \mathrm{H}]$ range of -0.05 to -0.15$)$ and metal-poor sub-regions $([\mathrm{Fe} / \mathrm{H}] \mathrm{range}$ of -0.65 to -0.95$)$ within the LMC are shown in red and green colours, respectively. For the SMC, the metal-rich sub-regions $([\mathrm{Fe} / \mathrm{H}]$ range of -0.60 to -0.85$)$ within the galaxy are shown as blue points. The remaining points in the two galaxies are shown as black dots. The centre of the two galaxies are shown as magenta-coloured diamonds. The centre of the SMC Wing is shown as magenta asterisk (Rubele et al. 2015). The main part of the MB is plotted as open squares (Skowron et al. 2014), as observed by the OGLE IV survey region.

We resorted to the same technique of RGB slope estimation for the LMC and the SMC. Also, we used results from CaT spectroscopy of RGs by the same group for both MCs [Cole et al. (2005), Grocholski et al. (2006) for the LMC and Dobbie et al. (2014b) for the $\mathrm{SMC}$ ] to calibrate the metallicity maps. Given that we expect only small systematic differences between the spectroscopic technique used for both galaxies, the similarity in the metallicity range of the metal-rich regions within the SMC and the metal-poor outliers within the LMC seems intriguing. On the other hand, given the values of mean metallicity of the LMC $(\approx-0.4 \mathrm{dex}$, Paper I) and the SMC $(\approx-0.95 \mathrm{dex}$, this work $)$ as well as the metallicity range within these galaxies, this similarity is not surprising. We suggest a spectroscopic and kinematic analysis of the metal-poor outliers within the LMC and the metal-rich regions within the SMC to check for any similarities between these regions. Obtaining highresolution, high-S/N spectra of stars within these areas would identify any commonalities or differences in the detailed distribution of abundances. The LMC metal-poor stars have a distinctive contribution from AGB-star winds to s-process elements and unusual elements, similar to alpha elements to the MW, but very different $\mathrm{Cu}$ abundances (Van der Swaelmen et al. 2013). It would be of great interest to see if those traits are shared in the SMC at the same metallicity.

\section{SUMMARY}

This paper presents an estimate of the average and radial variation of metallicity $([\mathrm{Fe} / \mathrm{H}])$ in the SMC based on photometric data. The results can be summarized as follows:

(i) We have successfully extended our technique of combining large-scale photometric data and spectroscopic data developed in
Paper I to estimate a metallicity map of the SMC. This again is a first of its kind high spatial resolution metallicity map for this particular galaxy derived using RGB stars from OGLE III and MCPS data sets.

(ii) We estimate the RGB slope of several sub-regions in the SMC and convert the slope to metallicity using spectroscopic data of Red Giants in the field.

(iii) The average metallicity of the SMC is found to be $-0.94 \mathrm{dex}$ $(\sigma[\mathrm{Fe} / \mathrm{H}]=0.09)$ from OGLE III data and $-0.95 \operatorname{dex}(\sigma[\mathrm{Fe} / \mathrm{H}]=$ 0.08 ) from MCPS data, within a radius of $2.5^{\circ}$.

(iv) Using these large-scale photometric data, we confirm once and for all that there exists an MG within the inner SMC, which is in agreement with previous spectroscopic results. The estimated MG using both data sets is shallow and gradual from the SMC centre till a radius $\sim 2.5^{\circ}$ : from $-0.045 \pm 0.004 \mathrm{dex} \mathrm{deg}^{-1}$ for MCPS to $-0.067 \pm 0.006 \mathrm{dex} \mathrm{deg}^{-1}$ for OGLE III).

(v) We find the MG within the SMC to be radially asymmetric.

(vi) The metallicity range of metal-rich regions around the SMC centre $(\approx-0.6$ to $-0.85 \mathrm{dex})$ is similar to that of metal-poor outliers located in the outskirts of the LMC. Such regions need to be studied in detail using spectroscopic studies to investigate the commonality.

\section{ACKNOWLEDGEMENTS}

This research was supported by the KASI-Yonsei Joint Research Program for all Frotiers of Astronomy and Space Science funded by the Korea Astronomy and Space Science Institute. The work is also partially supported by Basic Science Research Program through the National Research Foundation of Korea (NRF) funded by the Ministry of Education (NRF2016R1D1A1B01006608). S.C. acknowledges Dr. Smitha Subramanian (Kavli Institute for 
Astronomy and Astrophysics, Peking University, Beijing, China) for suggestions during initial area binning of OGLE III and MCPS data, and also for critical reading of the original version of the manuscript and comments. The authors thank the OGLE and the MCPS team for making the data available in public domain. The authors also thank the anonymous referee for constructive suggestions that helped improve the manuscript.

\section{REFERENCES}

Bekki K., Chiba M., 2008, ApJ, 679, L89

Besla G., Kallivayalil N., Hernquist L., Robertson B., Cox T. J., van der Marel R. P., Alcock C., 2007, AJ, 668, 949

Besla G., Kallivayalil N., Hernquist L., van der Marel R. P., Cox T. J., 2012, MNRAS, 421, 2109

Butler D., Demarque P., Smith H. A., 1982, ApJ, 257, 592

Carrera R., Gallart C., Aparicio A., Costa E., Méndez R. A., Noël N. E. D., 2008, AJ, 136, 1039

Choudhury S., Subramaniam A., Cole A. A., 2016, MNRAS, 455, 1855 (Paper I)

Cignoni M., Cole A. A., Tosi M., Gallagher J. S., Sabbi E., Anderson J., Grebel E. K., Nota A., 2013, ApJ, 775, 83

Cioni M.-R. L., 2009, A\&A, 506, 1137

Cole A. A., Tolstoy E., Gallagher J. S., III, Smecker-Hane T. A., 2005, AJ, 129,1465

Crowl H. H., Sarajedini A., Piatti A. E., Geisler D., Bica E., Clariá J. J., Santos J. F. C., Jr, 2001, AJ, 122, 220

Da Costa G. S., Armandroff T. E., 1990, AJ, 100, 162

Da Costa G. S., Hatzidimitriou D., 1998, AJ, 115, 1934

de Vaucouleurs G., Freeman K. C., 1972, Vistas in Astronomy, 14, 163

Deb S., Singh H. P., 2010, MNRAS, 402, 691

Deb S., Singh H. P., Kumar S., Kanbur S. M., 2015, MNRAS, 449, 2768

Dobbie P. D., Cole A. A., Subramaniam A., Keller S., 2014a, MNRAS, 442, 1663

Dobbie P. D., Cole A. A., Subramaniam A., Keller S., 2014b, MNRAS, 442, 1680

Evans C. J., Howarth I. D., 2008, MNRAS, 386, 826

Fujimoto M., Murai T., 1984, in van den Bergh S., de Boer K. S. D., eds, Proc. IAU Symp. 108, Structure and Evolution of the Magellanic Clouds. D. Reidel Publishing Co.. Dordrecht, p. 115

Gardiner L. T., Hawkins M. R. S., 1991, MNRAS, 251, 174

Gardiner L. T., Sawa T., Fujimoto M., 1994, MNRAS, 266, 567

Glatt K. et al., 2008a, AJ, 135, 1106

Glatt K. et al., 2008b, AJ, 136, 1703

Grocholski A. J., Cole A. A., Sarajedini A., Geisler D., Smith V. V., 2006, AJ, 132, 1630

Harris J., Zaritsky D., 2006, AJ, 131, 2514

Haschke R., Grebel E. K., Duffau S., 2012, AJ, 144, 107

Idiart T. P., Maciel W. J., Costa R. D. D., 2007, A\&A, 472, 101
Jacyszyn-Dobrzeniecka A. M. et al., 2016, AcA, 66, 149

Jacyszyn-Dobrzeniecka A. M. et al., 2017, AcA, 67, 1

Kapakos E., Hatzidimitriou D., 2012, MNRAS, 426, 2063

Kapakos E., Hatzidimitriou D., Soszyński I., 2011, MNRAS, 415, 1366

Kuchinski L. E., Frogel J. A., Terndrup D. M., Persson S. E., 1995, AJ, 109, 1131

Manning E. M., Cole A. A., 2017, MNRAS, 471, 4194

Mathewson D. S., Ford V. L., Visvanathan N., 1986, ApJ, 301, 664

Mathewson D. S., Ford V. L., Visvanathan N., 1988, ApJ, 333, 617

Murai T., Fujimoto M., 1980, PASJ, 32, 581

Muraveva T. et al., 2018, MNRAS, 473, 3131

Nidever D. L., Majewski S. R., Muñoz R. R., Beaton R. L., Patterson R. J., Kunkel W. E., 2011, ApJ, 733, L10

Olsen K. A. G., Zaritsky D., Blum R. D., Boyer M. L., Gordon K. D., 2011, ApJ, 737, 29

Parisi M. C., Grocholski A. J., Geisler D., Sarajedini A., Clariá J. J., 2009, AJ, 138, 517

Parisi M. C., Geisler D., Grocholski A. J., Clariá J. J., Sarajedini A., 2010, AJ, 139, 1168

Parisi M. C., Geisler D., Clariá J. J., Villanova S., Marcionni N., Sarajedini A., Grocholski A. J., 2015, AJ, 149, 154

Parisi M. C., Geisler D., Carraro G., Clariá J. J., Villanova S., Gramajo L. V., Sarajedini A., Grocholski A. J., 2016, AJ, 152, 58

Piatti A. E., 2011, MNRAS, 418, L69

Piatti A. E., 2012, MNRAS, 422, 1109

Piatti A. E., Sarajedini A., Geisler D., Clark D., Seguel J., 2007a, MNRAS, 377,300

Piatti A. E., Sarajedini A., Geisler D., Gallart C., Wischnjewsky M., 2007b, MNRAS, 381, L84

Ripepi V. et al., 2017, MNRAS, 472, 808

Rubele S. et al., 2015, MNRAS, 449, 639

Scowcroft V., Freedman W. L., Madore B. F., Monson A., Persson S. E., Rich J., Seibert M., Rigby J. R., 2016, ApJ, 816, 49

Skowron D. M. et al., 2014, ApJ, 795, 108

Subramanian S., Subramaniam A., 2009, A\&A, 496, 399

Subramanian S., Subramaniam A., 2012, ApJ, 744, 128

Subramanian S., Subramaniam A., 2015, A\&A, 573, A135

Subramanian S. et al., 2017, MNRAS, 467, 2980

Tanaka K. I., 1981, PASJ, 33, 247

Udalski A. et al., 2008, AcA, 58, 329

Van der Swaelmen M., Hill V., Primas F., Cole A. A., 2013, A\&A, 560, A44

Westerlund B. E., 1997, The Magellanic Clouds. Cambridge Univ. Press. Cambridge

Zaritsky D., Harris J., Grebel E. K., Thompson I. B., 2000, ApJ, 534, L53

Zaritsky D., Harris J., Thompson I. B., Grebel E. K., Massey P., 2002, AJ, 123,855

This paper has been typeset from a $\mathrm{T}_{\mathrm{E}} \mathrm{X} / \mathrm{LAT} \mathrm{E}$ file prepared by the author. 